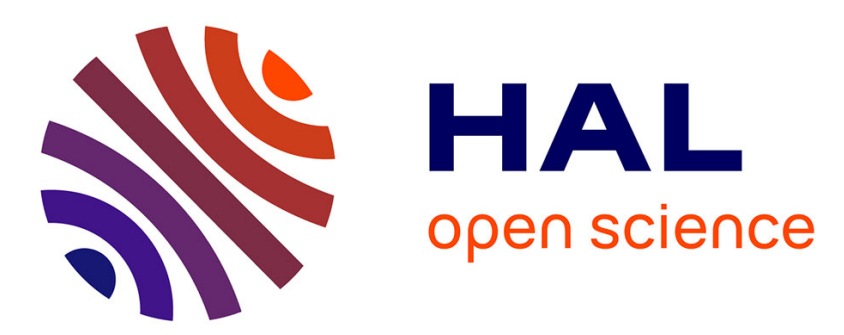

\title{
TOPOLOGICAL MIXING OF WEYL CHAMBER FLOWS
}

Nguyen-Thi Dang, Olivier Glorieux

\section{To cite this version:}

Nguyen-Thi Dang, Olivier Glorieux. TOPOLOGICAL MIXING OF WEYL CHAMBER FLOWS.

Ergodic Theory and Dynamical Systems, 2021, 41 (5), pp.1342-1368. hal-01877871

\section{HAL Id: hal-01877871 \\ https://hal.science/hal-01877871}

Submitted on 20 Sep 2018

HAL is a multi-disciplinary open access archive for the deposit and dissemination of scientific research documents, whether they are published or not. The documents may come from teaching and research institutions in France or abroad, or from public or private research centers.
L'archive ouverte pluridisciplinaire HAL, est destinée au dépôt et à la diffusion de documents scientifiques de niveau recherche, publiés ou non, émanant des établissements d'enseignement et de recherche français ou étrangers, des laboratoires publics ou privés. 


\title{
TOPOLOGICAL MIXING OF THE WEYL CHAMBER FLOW
}

\author{
NGUYEN-THI DANG, OLIVIER GLORIEUX
}

Abstract. In this paper, we study topological properties of the right action by translation of the Weyl Chamber flow on the space of Weyl chambers. We obtain a necessary and sufficient condition for topological mixing.

$\left({ }^{1}\right)$

\section{INTRODUCTION}

Let $G$ be semisimple real, connected, Lie group of non compact type. Let $K$ be a maximal compact subgroup of $G$ and $A$ a maximal torus of $G$ for which there is a Cartan decomposition. Let $M$ be the centralizer of $A$ in $K$. We establish mixing properties for right action by translation of one parameter subgroups of $A$ on quotients $\Gamma \backslash G / M$ where $\Gamma$ is a discrete, Zariski dense subgroup of $G$.

The particular case when $G$ is of real rank one is well known. In this case, the symmetric space $X=G / K$ is a complete, connected, simply connected Riemannian manifold of negative curvature. The right action by translation of $A$ on $G / M$ coincides with the geodesic flow on $T^{1} X$. Dal'bo [Dal00] proved that it is mixing (on its nonwandering set) if and only if the length spectrum is non arithmetic. The latter holds when $\Gamma$ is a Zariski dense subgroup, see Benoist [Ben00], Kim [Kim06].

We are interested in cases where $G$ is of higher real rank $k \geq 2$. When $\Gamma \backslash G / M$ is of finite volume, i.e. when $\Gamma$ is a lattice, it follows from Howe-Moore's Theorem that the action of any noncompact subgroup of $G$ is mixing.

We study the general situation of any discrete, Zariski dense subgroup, which of course includes the case of lattices.

If $\Gamma \backslash G / M$ has infinite volume, the known results are not as general.

In the particular case of so-called Ping-Pong subgroups of $\operatorname{PSL}(k+1, \mathbb{R})$, Thirion [Thi07], [Thi09] proved mixing with respect to a natural measure on $\Omega(X)$ for a one parameter flow associated to the "maximal growth vector" introduced by Quint in [Qui02]. Sambarino [Sam15] did the same for hyperconvex representations.

Finally, Conze-Guivarc'h in [CG02] proved for any Zariski dense subgroup $\Gamma$, the topological transitivity (i.e. existence of dense orbits) of the right $A$-action on a natural closed $A M$-invariant set $\Omega(X) \subset \Gamma \backslash G / M$.

Let $\mathfrak{a} \simeq \mathbb{R}^{k}$ be the Cartan Lie subalgebra over $A$ and $\mathfrak{a}^{++}$the choice of a positive Weyl chamber. For any $\theta \in \mathfrak{a}^{++}$, the Weyl chamber flow $\left(\phi_{t}^{\theta}\right)$ corresponds to the right action by translation of $\exp (t \theta)$. Benoist [Ben97] introduced a convex limit cone $\mathcal{C}(\Gamma) \subset \mathfrak{a}$ and proved that for Zariski dense semigroups, the limit cone is of non empty interior. We prove topological mixing for any direction of the interior of $\mathcal{C}(\Gamma)$.

\footnotetext{
${ }^{1}$ MSC Classification 54H20, 37B99, 53C30, 58E40 Secondary 53C35
} 
Theorem 1.1. Let $G$ be a semisimple, connected, real linear Lie group, of non-compact type. Let $\Gamma$ be a Zariski dense, discrete subgroup of $G$. Let $\theta \in \mathfrak{a}^{++}$.

Then the dynamical system $\left(\Omega(X), \phi_{t}^{\theta}\right)$ is topologically mixing if and only if $\theta$ is in the interior of the limit cone $\mathcal{C}(\Gamma)$.

Taking $\widetilde{\Omega} \subset G / M$ to be the universal cover of $\Omega(X)$, we remark that this Theorem is a direct consequence of the following statement, where $\Gamma$ is a Zariski dense semigroup of $G$. We insist that under this hypothesis, $\Gamma$ is not necessarily a subgroup and can even be non discrete.

Theorem 1.2. Let $G$ be a semisimple, connected, real linear Lie group, of non-compact type. Let $\Gamma$ be a Zariski dense semigroup of $G$. Let $\theta \in \mathfrak{a}^{++}$.

Then $\theta$ is in the interior of the limit cone if and only if for all nonempty open subsets $\widetilde{U}, \widetilde{V} \subset$ $\widetilde{\Omega}(X)$, there exists $T>0$ so that for any later time $t>T$, there exists $\gamma_{t} \in \Gamma$ with

$$
\gamma_{t} \widetilde{U} \cap \phi_{t}^{\theta}(\widetilde{V}) \neq \emptyset \text {. }
$$

In the first section, we give some background on globally symmetric spaces. We introduce the space of Weyl chambers, the Weyl chamber flow, give a compactification of the space of Weyl chambers and present a higher rank generalization of the Hopf coordinates.

In the second section, we introduce the main tools: Schottky semigroups and estimations on the spectrum of products of elements in $G$.

In the third section, we introduce the non-wandering Weyl chambers set, it is a closed $A M$-invariant subset $\Omega(X) \subset \Gamma \backslash G / M$. Then we study topological transitivity in Proposition 4.7. We prove that if the flow $\phi_{t}^{\theta}$ is topologically transitive in $\Omega(X)$, where $\theta \in \mathfrak{a}^{++}$, then the direction $\theta$ must be in the interior of the limit cone. Since topological mixing implies topological transitivity, this provides one direction of the main Theorem 1.2.

In the last section, we prove a key Proposition 5.4 using density results that come from nonarithmeticity of the length spectrum. Then we prove the main theorem.

In the appendix we prove a density lemma of subgroups of $\mathbb{R}^{n}$ needed in the proof of Proposition 5.4.

In the whole article, $G$ is a semisimple, connected, real linear Lie group, of non-compact type.

\section{BACKGROUND ON SYMMETRIC SPACES}

Classical references for this section are [Thi07, Chapter 8, §8.B, 8.D, 8.E, 8,G], [GJT12, Chapter III, §1-4] and [Hel78, Chapter IV, Chapter V, Chapter VI].

Let $K$ be a maximal compact subgroup of $G$. Then $X=G / K$ is a globally symmetric space of non-compact type. The group $G$ is the identity component of its isometry group. It acts transitively on $X$, by left multiplication. We fix a point $o=K \in X$. Then $K$ is in the fixed point set of the involutive automorphism induced by the geodesic symmetry in $o$ (cf. [Hel78, Chapter VI, Thm 1.1]).

Denote by $\mathfrak{g}$ (resp. $\mathfrak{k})$ the Lie algebra of $G$ (resp. $K$ ). The differential of the involutive automorphism induced by the geodesic symmetry in $o$ is a Cartan involution of $\mathfrak{g}$. Then $\mathfrak{k}$ is the eigenspace of the eigenvalue 1 (for the Cartan involution) and we denote by $\mathfrak{p}$ the eigenspace of the eigenvalue -1 . The decomposition $\mathfrak{g}=\mathfrak{k} \oplus \mathfrak{p}$ is a Cartan decomposition.

2.1. Flats, Weyl Chambers, classical decompositions. A flat of the symmetric space $X$ is a totally geodesic, isometric embedding of a Euclidean space. We are interested in flats of maximal dimension in $X$, called maximal flats. One can construct the space of maximal flats following [Thi07, Chapter 8, §8.D, 8,D] thanks to [Hel78, Chapter V, Prop. 6.1]. Let $\mathfrak{a} \subset \mathfrak{p}$ be a Cartan subspace of $\mathfrak{g}$ i.e. a maximal abelian subspace such that the adjoint endomorphism 
of every element is semisimple. We denote by $A$ the subgroup $\exp (\mathfrak{a})$. The real rank of the symmetric space $X$, denoted by $r_{G}$, is the dimension of the real vector space $\mathfrak{a}$.

Definition 2.1. A parametrized flats is an embedding of $\mathfrak{a}$ of the form $g f_{0}$, where $g \in G$ and $f_{0}$ is the map defined by

$$
\begin{aligned}
f_{0}: \mathfrak{a} & \longrightarrow X \\
v & \longmapsto \exp (v)_{o} .
\end{aligned}
$$

We denote by $\mathcal{W}(X)$ the set of parametrized flats of $X$.

By definition, the set of parametrized flats is the orbit of $f_{0}$ under the left-action by multiplication of $G$. The stabilizer of $f_{0}$ is the centralizer of $A$ in $K$, denoted by $M$. We deduce that the set of parametrized flats $\mathcal{W}(X)$ identifies with the homogeneous space $G / M$. For any parametrized flat $f \in \mathcal{W}(X)$, there is an element $g_{f}$ in $G$ such that $f=g_{f} f_{0}$. Hence, the map

$$
\begin{aligned}
\mathcal{W}(X) & \stackrel{\sim}{\longrightarrow} G / M \\
f & \longmapsto g_{f} M
\end{aligned}
$$

is a $G$-equivariant homeomorphism.

For any linear form $\alpha$ on $\mathfrak{a}$, set $\mathfrak{g}_{\alpha}:=\{v \in \mathfrak{g} \mid \forall u \in \mathfrak{a},[u, v]=\alpha(u) v\}$. The set of restricted roots is $\Sigma:=\left\{\alpha \in \mathfrak{a}^{*} \backslash\{0\} \mid \mathfrak{g}_{\alpha} \neq\{0\}\right\}$. The kernel of each restricted root is a hyperplane of $\mathfrak{a}$. The Weyl Chambers of $\mathfrak{a}$ are the connected components of $\mathfrak{a} \backslash \cup_{\alpha \in \Sigma} \operatorname{ker}(\alpha)$. We fix such a component, call it the positive Weyl chamber and denote it (resp. its closure) by $\mathfrak{a}^{++}$(resp. $\mathfrak{a}^{+}$).

We denote by $N_{K}(A)$ the normalizer of $A$ in $K$. The group $N_{K}(A) / M$ is called the Weyl group. The positive Weyl chamber of $\mathfrak{a}$ allows us to tesselate the maximal flats in the symmetric space $X$. Indeed, $f_{0}\left(\mathfrak{a}^{+}\right)$is a fundamental domain for the action of the Weyl group on the maximal flat $f_{0}(\mathfrak{a})$ and $G$ acts transitively on the space of parametrized flats. Finally, the orbit G. $f_{0}\left(\mathfrak{a}^{+}\right)$identifies with the space of parametrized flats, the image of $g . f_{0}\left(\mathfrak{a}^{+}\right)$is a geometric Weyl chamber. This explains why the set of parametrized flats is also called the space of Weyl chambers. For any geometric Weyl chamber $f\left(\mathfrak{a}^{+}\right) \in G . f_{0}\left(\mathfrak{a}^{+}\right)$, the image of $0 \in \mathfrak{a}^{+}$is the origin. Furthermore,

$$
G / M \simeq \mathcal{W}(X) \simeq G \cdot f_{0}\left(\mathfrak{a}^{+}\right) .
$$

Definition 2.2. The right-action of $\mathfrak{a}$ on $\mathcal{W}(X)$ is defined by $\alpha \cdot f: v \mapsto f(v+\alpha)$ for all $\alpha \in \mathfrak{a}$ and $f \in \mathcal{W}(X)$. The Weyl Chamber Flow, is defined for all $\theta \in \mathfrak{a}_{1}^{++}$and $f \in \mathcal{W}(X)$ by

$$
\begin{aligned}
\phi^{\theta}(f): \mathbb{R} & \longrightarrow \mathcal{W}(X) \\
t & \longmapsto \phi_{t}^{\theta}(f)=f(v+\theta t)=f(v) e^{\theta t} .
\end{aligned}
$$

Remark that the Weyl Chamber Flow $\phi_{t}^{\theta}$ is also the right-action of the one-parameter subgroup $\exp (t \theta)$ on the space of Weyl chambers.

The set of positive roots, denoted by $\Sigma^{+}$, is the subset of roots which take positive values in the positive Weyl chamber. The positive Weyl chamber also allows to define two particular nilpotent subalgebras $\mathfrak{n}=\oplus_{\alpha \in \Sigma^{+}} \mathfrak{g}_{\alpha}$ and $\mathfrak{n}^{-}=\oplus_{\alpha \in \Sigma^{+}} \mathfrak{g}_{-\alpha}$. Finally, set $A^{+}:=\exp \left(\mathfrak{a}^{+}\right), A^{++}:=\exp \left(\mathfrak{a}^{++}\right)$, $N:=\exp (\mathfrak{n})$ and $N^{-}:=\exp \left(\mathfrak{n}^{-}\right)$. For all $a \in A^{++}, h_{+} \in N, h_{-} \in N^{-}$notice that

$$
a^{-n} h_{ \pm} a^{n} \underset{ \pm \infty}{\longrightarrow} i d_{G} .
$$

Definition 2.3. For any $g \in G$, we define, by Cartan decomposition, a unique element $\mu(g) \in \mathfrak{a}^{+}$ such that $g \in K \exp (\mu(g)) K$. The map $\mu: G \rightarrow \mathfrak{a}^{+}$is called the Cartan projection. 
The Cartan projection allows to define an $\mathfrak{a}^{+}$-valued function on $X \times X$, denoted by $d_{\mathfrak{a}^{+}}$, following [Thi07, Def-Thm 8.38]. For any $x, x^{\prime} \in X$, there exists $g, g^{\prime} \in G$ so that $x=g K$ and $x^{\prime}=g^{\prime} K$, we set

$$
d_{\mathfrak{a}^{+}}\left(x, x^{\prime}\right):=\mu\left(g^{\prime-1} g\right) .
$$

This function is independent of the choice of $g$ and $g^{\prime}$. Recall [Hel78, Chapter V, Lemma 5.4] that $\mathfrak{a}$ is endowed with a scalar product coming from the Killing form on $\mathfrak{g}$, and the norm of $d_{\mathfrak{a}^{+}}\left(x, x^{\prime}\right)$ coincides with the distance between $x$ and $x^{\prime}$ in the symmetric space $X$.

An element of $G$ is unipotent if all its eigenvalues are equal to 1 and equivalently if it is the exponential of a nilpotent element. An element of $G$ is semisimple if it is diagonalizable over $\mathbb{C}$, elliptic (resp. hyperbolic) if it is semisimple with eigenvalues of modulus 1 (resp. real eigenvalues). Equivalently, elliptic (resp. hyperbolic, unipotent) elements are conjugated to elements in $K$ (resp. $A, N)$.

Any element $g \in G$ admits a unique decomposition (in $G$ ) $g=g_{e} g_{h} g_{u}$, called the Jordan decomposition, where $g_{e}, g_{h}$ and $g_{u}$ commute and where $g_{e}$ (resp. $g_{h}, g_{u}$ ) is elliptic (resp. hyperbolic, unipotent). The element $g_{e}$ (resp. $\left.g_{h}, g_{u}\right)$ is called the elliptic part (resp. hyperbolic part, unipotent part) of $g$.

Definition 2.4. For any element $g \in G$, there is a unique element $\lambda(g) \in \mathfrak{a}^{+}$such that the hyperbolic part of $g$ is conjugated to $\exp (\lambda(g)) \in A^{+}$. The map $\lambda: G \rightarrow \mathfrak{a}^{+}$is called the Jordan projection.

An element $g \in G$ is loxodromic if $\lambda(g) \in \mathfrak{a}^{++}$. Since any element of $N$ that commute with $\mathfrak{a}^{++}$ is trivial, the unipotent part of loxodromic elements is trivial. Furthermore, the only elements of $K$ that commute with $\mathfrak{a}^{++}$are in $M$. We deduce that the elliptic part of loxodromic elements are conjugated to elements in $M$. Hence, for any loxodromic element $g \in G$, there exists $h_{g} \in G$ and $m(g) \in M$ so that we can write $g=h_{g} m(g) e^{\lambda(g)} h_{g}^{-1}$. For any $m \in M$ we can also write $g=\left(h_{g} m\right)\left(m^{-1} m(g) m\right) e^{\lambda(g)}\left(h_{g} m\right)^{-1}$. This allows us to associate to any loxodromic element $g \in G$, an angular part $m(g)$ which is defined up to conjugacy by $M$.

The spectral radius formula [BQ16, Corollary 5.34]

$$
\lambda(g)=\lim _{n \rightarrow \infty} \frac{1}{n} \mu\left(g^{n}\right)
$$

allows to compute the Jordan projection thanks to the Cartan projection.

Definition 2.5. For any $g \in G$, there exists a unique triple $(k, v, n) \in K \times \mathfrak{a} \times N$ such that $g=k \exp (v) n$. Furthermore, the map

$$
\begin{aligned}
K \times \mathfrak{a} \times N & \longrightarrow G \\
(k, v, n) & \longmapsto k e^{v} n
\end{aligned}
$$

is a diffeomorphism called the Iwasawa decomposition.

2.2. Asymptotic Weyl chambers, Busemann-Iwasawa cocycle. The main references for this subsection are [Thi07, Chapter 8, §8.D], [GJT12] and [BQ16].

We endow the space of geometric Weyl chambers with the equivalence relation

$$
f_{1}\left(\mathfrak{a}^{+}\right) \sim f_{2}\left(\mathfrak{a}^{+}\right) \Leftrightarrow \sup _{u \in \mathfrak{a}^{++}} d\left(f_{1}(u), f_{2}(u)\right)<\infty .
$$

Equivalently, $f_{1}\left(\mathfrak{a}^{+}\right) \sim f_{2}\left(\mathfrak{a}^{+}\right)$if and only if for any $v \in \mathfrak{a}^{++}$, the geodesics $t \mapsto f_{1}(t v)$ and $t \mapsto f_{2}(t v)$ are at bounded distance when $t \rightarrow+\infty$. Equivalence classes for this relation are called asymptotic Weyl chambers. We denote by $\mathcal{F}(X)$ the set of asymptotic Weyl chambers and by $\eta_{0}$ the asymptotic class of the Weyl chamber $f_{0}\left(\mathfrak{a}^{+}\right)$. 
Fact 2.6. The set $\mathcal{F}(X)$ identifies with the Furstenberg boundary $G / P$ where $P=M A N$. Furthermore

$$
G / P \simeq \mathcal{F}(X) \simeq K / M \simeq K \cdot \eta_{0} .
$$

Proof. Since $G$ acts transitively on the space of Weyl chambers, it also acts transitively on the set of asymptotic Weyl chambers.

We show that $P$ is the stabilizer of $\eta_{0}$. For any $g \in G$ and $u \in \mathfrak{a}^{++}$, we compute the distance

$$
d\left(g f_{0}(u), f_{0}(u)\right)=\left\|d_{\mathfrak{a}^{+}}\left(g f_{0}(u), f_{0}(u)\right)\right\|=\left\|\mu\left(e^{-u} g e^{u}\right)\right\| .
$$

By Bruhat decomposition (see [Hel78, Chapter IX, Thm 1.4]), there exists an element $w$ in the normalizer of $A$ in $K$ and elements $p_{1}, p_{2} \in P=M A N$ so that $g=p_{1} w p_{2}$. Then

$$
e^{-u} g e^{u}=\left(e^{-u} p_{1} e^{u}\right) e^{-u}\left(w e^{u} w^{-1}\right) w\left(e^{-u} p_{2} e^{u}\right) .
$$

Note that by equation (1), the sets $\left\{e^{-u} p_{i} e^{u}\right\}_{u \in \mathfrak{a}^{++}, i=1,2}$ are bounded. Hence, the sets $\left\{e^{-u} g e^{u}\right\}_{u \in \mathfrak{a}^{++}}$ and $\left\{e^{-u} w e^{u} w^{-1}\right\}_{u \in \mathfrak{a}^{++}}$have the same behavior. Remark now that $e^{-u} w e^{u} w^{-1}=e^{-u+A d(w) u}$, which is bounded uniformly in $\mathfrak{a}^{++}$only when $w \in M$. We deduce that $\left\{e^{-u} g e^{u}\right\}_{u \in \mathfrak{a}^{++}}$is bounded only when $g \in P$. Hence the subgroup $P$ is the stabilizer of the asymptotic class $\eta_{0}$.

The geometric Weyl chambers whose origin is $o \in X$ are in the orbit $K \cdot f_{0}\left(\mathfrak{a}^{+}\right)$. Any equivalence class in $\mathcal{F}(X)$ admits, by Iwasawa decomposition, a unique representative in $K . f_{0}\left(\mathfrak{a}^{+}\right)$. Moreover, $K / M$ identifies with the orbit $K . f_{0}\left(\mathfrak{a}^{+}\right)$since $M$ is the stabilizer of $f_{0}$ in $K$.

For any asymptotic Weyl chamber $\eta \in \mathcal{F}(X)$ and $g \in G$, consider, by Iwasawa decomposition, the unique element $\sigma(g, \eta) \in \mathfrak{a}$, called the Iwasawa cocycle, such that if $k_{\eta} \in K$ satisfies $\eta=k_{\eta} \eta_{0}$, then

$$
g k_{\eta} \in K \exp (\sigma(g, \eta)) N .
$$

The cocycle relation holds (cf [BQ16, Lemma 5.29]) i.e. for all $g_{1}, g_{2} \in G$ and $\eta \in \mathcal{F}(X)$ then

$$
\sigma\left(g_{1} g_{2}, \eta\right)=\sigma\left(g_{1}, g_{2} \eta\right)+\sigma\left(g_{2}, \eta\right) .
$$

For any pair of points $x, y \in X$, any asymptotic Weyl chamber $\eta \in \mathcal{F}(X)$ and $u \in \mathfrak{a}^{++}$, we consider a representative $f_{\eta}\left(\mathfrak{a}^{+}\right)$of $\eta$ and define the Busemann cocycle by

$$
\beta_{f_{\eta}, u}(x, y)=\lim _{t \rightarrow+\infty} d_{\mathfrak{a}^{+}}\left(f_{\eta}(t u), x\right)-d_{\mathfrak{a}^{+}}\left(f_{\eta}(t u), y\right) .
$$

It turns out that the Busemann cocycle depends neither on the choice of the geometric Weyl chamber in the class $\eta$, nor on the choice of $u \in \mathfrak{a}^{++}$. We will write $\beta_{f_{\eta}, u}(x, y)=\beta_{\eta}(x, y)$. By [BQ16, Corollary 5.34], the Iwasawa and Busemann cocycle coincide in the sense that for all $g \in G, \eta \in \mathcal{F}(X)$ and $u \in \mathfrak{a}^{++}$,

$$
\beta_{f_{\eta}, u}\left(g^{-1} o, o\right)=\sigma(g, \eta) .
$$

We associate attractive and repulsive asymptotic geometric Weyl chambers to loxodromic elements of $G$ as follows.

Recall that for any loxodromic element $g \in G$, there is an element $h_{g} \in G$ and an angular part $m(g) \in M$ so that $g=h_{g} e^{\lambda(g)} m(g) h_{g}^{-1}$. We set $g^{+}:=\left[h_{g} \cdot f_{0}\left(\mathfrak{a}^{+}\right)\right]$and $g^{-}:=\left[h_{g} \cdot f_{0}\left(-\mathfrak{a}^{+}\right)\right]$. Then $g^{+} \in \mathcal{F}(X)$ (resp. $g^{-}$) is called the attractive (resp. repulsive) asymptotic Weyl chamber.

Fact 2.7. For any loxodromic element $g \in G$, we have $\lambda(g)=\sigma\left(g, g^{+}\right)$.

Proof. Let $g \in G$ be a loxodromic element. Consider an element $h_{g} \in G$ and an angular part $m(g) \in M$ so that $g=h_{g} e^{\lambda(g)} m(g) h_{g}^{-1}$. Denote by $f_{g}$ the parametrized flat $f_{g}: v \mapsto h_{g} e^{v} o$. Then the geometric Weyl chamber $f_{g}\left(\mathfrak{a}^{+}\right)$(resp. $f_{g}\left(-\mathfrak{a}^{+}\right)$) is a representative of the limit points $g^{+}\left(\right.$resp. $\left.g^{-}\right)$. 
Fix any $u \in \mathfrak{a}^{++}$. Then by equation (2), we deduce

$$
\begin{aligned}
\sigma\left(g, g^{+}\right)=\beta_{f_{g}, u}\left(g^{-1} o, o\right) & =\lim _{t \rightarrow+\infty} d_{\mathfrak{a}^{+}}\left(f_{g}(t u), g^{-1} o\right)-d_{\mathfrak{a}^{+}}\left(f_{g}(t u), o\right) \\
& =\lim _{t \rightarrow+\infty} \mu\left(g h_{g} e^{t u}\right)-\mu\left(h_{g} e^{t u}\right) \\
& =\lim _{t \rightarrow+\infty} \mu\left(h_{g} e^{\lambda(g)+t u} m(g)\right)-\mu\left(h_{g} e^{t u}\right) .
\end{aligned}
$$

By left and right $K$-invariance of the Cartan projection, we deduce that $\mu\left(h_{g} e^{\lambda(g)+t u} m(g)\right)=$ $\mu\left(h_{g} e^{\lambda(g)+t u}\right)$. Hence

$$
\sigma\left(g, g^{+}\right)=\lim _{t \rightarrow+\infty} \mu\left(h_{g} e^{\lambda(g)+t u}\right)-\mu\left(h_{g} e^{t u}\right) .
$$

By Iwasawa decomposition on $h_{g}$, there exists a unique unipotent element $n \in N$ so that $h_{g} \in$ $K e^{\sigma\left(h_{g}, \eta_{0}\right)} n$. Hence, for all $t \in \mathbb{R}_{+}$,

$$
\begin{aligned}
& \mu\left(h_{g} e^{\lambda(g)+t u}\right)-\mu\left(h_{g} e^{t u}\right)= \mu\left(e^{\sigma\left(h_{g}, \eta_{0}\right)} n e^{\lambda(g)+t u}\right) \\
&-\mu\left(e^{\sigma\left(h_{g}, \eta_{0}\right)} n e^{t u}\right) \\
&=\mu\left(e^{\sigma\left(h_{g}, \eta_{0}\right)+\lambda(g)+t u} e^{-\lambda(g)-t u} n e^{\lambda(g)+t u}\right) \\
&-\mu\left(e^{\sigma\left(h_{g}, \eta_{0}\right)+t u} e^{-t u} n e^{t u}\right) .
\end{aligned}
$$

Since $u \in \mathfrak{a}^{++}$, then for any $t \in \mathbb{R}_{+}$large enough, $\sigma\left(h_{g}, \eta_{0}\right)+\lambda(g)+t u$ and $\sigma\left(h_{g}, \eta_{0}\right)+t u$ are in $\mathfrak{a}^{+}$. Furthermore, by equation (1), we deduce

$$
\lim _{t \rightarrow+\infty} e^{-\lambda(g)-t u} n e^{\lambda(g)+t u}=\lim _{t \rightarrow+\infty} e^{-t u} n e^{t u}=i d_{G}
$$

Hence, by continuity of the Cartan projection, when $t \rightarrow+\infty$,

$$
\begin{aligned}
\mu\left(e^{\sigma\left(h_{g}, \eta_{0}\right)+\lambda(g)+t u} e^{-\lambda(g)-t u} n e^{\lambda(g)+t u}\right) & =\mu\left(e^{\sigma\left(h_{g}, \eta_{0}\right)+\lambda(g)+t u}\right)+o(1) \\
\mu\left(e^{\sigma\left(h_{g}, \eta_{0}\right)+t u} e^{-t u} n e^{t u}\right) & =\mu\left(e^{\sigma\left(h_{g}, \eta_{0}\right)+t u}\right)+o(1),
\end{aligned}
$$

and,

$$
\begin{aligned}
d_{\mathfrak{a}^{+}}\left(f_{g}(t u), g^{-1} o\right)-d_{\mathfrak{a}^{+}}\left(f_{g}(t u), o\right) & =\sigma\left(h_{g}, \eta_{0}\right)+\lambda(g)+t u-\left(\sigma\left(h_{g}, \eta_{0}\right)+t u\right)+o(1) \\
& =\lambda(g)+o(1) .
\end{aligned}
$$

Finally, $\lambda(g)=\sigma\left(g, g^{+}\right)$.

2.3. Hopf parametrization. Our main reference for this subsection is [Thi07, Chapter 8, $\S 8 . G .2]$.

In the geometric compactification of symmetric spaces of non-compact type, any bi-infinite geodesic defines opposite points in the geometric boundary. In a similar way, we introduce asymptotic Weyl chambers in general position.

We endow the product $\mathcal{F}(X) \times \mathcal{F}(X)$ with the diagonal left $G$-action. For any $(\xi, \eta) \in$ $\mathcal{F}(X) \times \mathcal{F}(X)$ and $g \in G$, we set $g \cdot(\xi, \eta):=(g . \xi, g \cdot \eta)$. For any parametrized flat $f \in \mathcal{W}(X)$, denote by $f_{+}$(resp. $f_{-}$) the asymptotic class of the geometric Weyl chamber $f\left(\mathfrak{a}^{+}\right)$(resp. $\left.f\left(-\mathfrak{a}^{+}\right)\right)$. Then the following map

$$
\begin{aligned}
\mathcal{H}^{(2)}: \mathcal{W}(X) & \longrightarrow \mathcal{F}(X) \times \mathcal{F}(X) \\
f & \longmapsto\left(f_{+}, f_{-}\right)
\end{aligned}
$$

is $G$-equivariant and continuous.

Two asymptotic Weyl chambers $\xi, \eta \in \mathcal{F}(X)$ are in general position or opposite, if they are in the image $\mathcal{H}^{(2)}(\mathcal{W}(X))$ i.e. if there exists a parametrized flat $f \in \mathcal{W}(X)$ such that the geometric Weyl chamber $f_{+}$(resp. $\left.f_{-}\right)$is a representative of $\xi$ (resp. $\eta$ ). 
We denote by $\mathcal{F}^{(2)}(X)$ the set of asymptotic Weyl chambers in general position. The product topology on the product space $\mathcal{F}(X) \times \mathcal{F}(X)$ induces a natural topology on $\mathcal{F}^{(2)}(X)$.

Fact 2.8 ( $\$ 3.2\left[\right.$ Thi09]). The set $\mathcal{F}^{(2)}(X)$ identifies with the homogeneous space $G / A M$. Furthermore, if we denote by $\eta_{0}$ (resp. $\check{\eta}_{0}$ ) the asymptotic class of the Weyl chamber $f_{0}\left(\mathfrak{a}^{+}\right)$(resp. $f_{0}\left(-\mathfrak{a}^{+}\right)$), then

$$
G .\left(\eta_{0}, \check{\eta}_{0}\right) \simeq \mathcal{F}^{(2)}(X) \simeq G / A M .
$$

The Hopf coordinates map is defined by

$$
\begin{aligned}
\mathcal{W}(X) & \longrightarrow \mathcal{F}^{(2)}(X) \times \mathfrak{a} \\
f & \longmapsto\left(f_{+}, f_{-} ; \beta_{f_{+}}(f(0), o)\right) .
\end{aligned}
$$

We define the left $G$-action on the skew product $\mathcal{F}^{(2)}(X) \times \mathfrak{a}$ as follows. For any $g \in G$ and $(\xi, \eta ; v) \in \mathcal{F}^{(2)}(X) \times \mathfrak{a}$, we set

$$
g .(\xi, \eta ; v)=\left(g \cdot \xi, g . \eta ; v+\beta_{g \cdot \xi}(g . o, o)\right) .
$$

The right $\mathfrak{a}$-action defined for any $\alpha \in \mathfrak{a}$ and $(\xi, \eta ; v) \in \mathcal{F}^{(2)}(X) \times \mathfrak{a}$ by

$$
\alpha \cdot(\xi, \eta ; v)=(\xi, \eta ; v+\alpha)
$$

is called the right $\mathfrak{a}-$ action by translation.

Similarly, for any $\theta \in \mathfrak{a}_{1}^{++}$, we define the Weyl chamber flow $\phi^{\theta}$ on the skew product, for all $(\xi, \eta ; v) \in \mathcal{F}^{(2)}(X) \times \mathfrak{a}$ and $t \in \mathbb{R}_{+}$,

$$
\phi_{t}^{\theta}(\xi, \eta ; v)=(\xi, \eta ; v+\theta t) .
$$

Proposition 2.9 (Proposition 8.54 [Thi07]). The Hopf coordinates map is a $(G, \mathfrak{a})$-equivariant homeomorphism in the sense that:

(i) The left-action of $G$ on $\mathcal{W}(X)$ identifies, via the Hopf coordinates map, with the left $G$-action on the skew product $\mathcal{F}^{(2)}(X) \times \mathfrak{a}$;

(ii) The right-action of $\mathfrak{a}$ on $\mathcal{W}(X)$ identifies, via the Hopf coordinates map, with the right $\mathfrak{a}-$ action by translation on the skew product $\mathcal{F}^{(2)}(X) \times \mathfrak{a}$.

Furthermore, for any $\theta \in \mathfrak{a}_{1}^{++}$and $t \in \mathbb{R}_{+}$, for all $f \in \mathcal{W}(X)$, we obtain

$$
\mathcal{H}\left(\phi_{t}^{\theta}(f)\right)=\phi_{t}^{\theta}(\mathcal{H}(f)) .
$$

\section{LOXODROMIC ELEMENTS}

We first study loxodromic elements in $G L(V)$ for $V$ a real vector space of finite dimension endowed with a Euclidean norm $\|$.$\| . Then we give some background on representations of$ semisimple Lie groups. Finally, we study the dynamical properties of the representations of $G$ acting on the projective space of those representations.

3.1. Proximal elements of $\operatorname{GL}(V)$. Denote by $X=\mathbb{P}(V)$ the projective space of $V$. We endow $X$ with the distance

$$
d(\mathbb{R} x, \mathbb{R} y)=\inf \left\{\left\|v_{x}-v_{y}\right\| \mid\left\|v_{x}\right\|=\left\|v_{y}\right\|=1, v_{x} \in \mathbb{R} x, v_{y} \in \mathbb{R} y\right\} .
$$

For $g \in \operatorname{End}(V)$, denote by $\lambda_{1}(g)$ its spectral radius.

Definition 3.1. An element $g \in \operatorname{End}(V) \backslash\{0\}$ is proximal on $X$ if it has a unique eigenvalue $\alpha \in \mathbb{C}$ such that $|\alpha|=\lambda_{1}(g)$ and this eigenvalue is simple (therefore $\alpha$ is a real number). Denote by $V_{+}(g)$ the one dimensional eigenspace corresponding to $\alpha$ and $V_{-}(g)$ the supplementary $g$ invariant hyperplane. In the projective space, denote by $x_{+}(g)=\mathbb{P}\left(V_{+}(g)\right)$ (resp. $X_{-}(g)=$ $\left.\mathbb{P}\left(V_{-}(g)\right)\right)$ the attractive point (resp. the repulsive hyperplane). 
The open ball centered in $x \in X$ of radius $\varepsilon>0$ is denoted by $B(x, \varepsilon)$. For every subset $Y \subset X$, we denote by $\mathcal{V}_{\varepsilon}(Y)$ the open $\varepsilon$-neighbourhood of $Y$. The following definition gives uniform control over the geometry of proximal elements (parametrized by $r$ ) and their contracting dynamics (parametrized by $\varepsilon$ ).

Definition 3.2. Let $0<\varepsilon \leq r$. A proximal element $g$ is $(r, \varepsilon)$-proximal if $d\left(x_{+}(g), X_{-}(g)\right) \geq 2 r$, $g$ maps $\mathcal{V}_{\varepsilon}\left(X_{-}(g)\right)^{c}$ into the ball $B\left(x_{+}(g), \varepsilon\right)$ and its restriction to the subset $\mathcal{V}_{\varepsilon}\left(X_{-}(g)\right)^{c}$ is an $\varepsilon$-Lipchitz map.

We give three remarks that follow from the definition.

1) If an element is $(r, \varepsilon)$-proximal, then it is $\left(r^{\prime}, \varepsilon\right)$-proximal for $\varepsilon \leq r^{\prime} \leq r$,

2) If an element is $(r, \varepsilon)$-proximal, then it is $\left(r, \varepsilon^{\prime}\right)$-proximal for $r \geq \epsilon^{\prime} \geq \varepsilon$.

3 ) If $g$ is is $(r, \varepsilon)$-proximal, then $g^{n}$ is also is $(r, \varepsilon)$-proximal for $n \geq 1$.

The numbers $r$ and $\varepsilon$ depend on the metric of the projective space, which, in our case, depends on the choice of the norm on the finite dimensional vector space. However, in [Ser16, Remark 2.3] Sert claims the following statement. We provide a proof for completeness.

Lemma 3.3. For every proximal transformation $g$, there exists $r>0$ and $n_{0} \in \mathbb{N}$ such that for all $n \geq n_{0}$ large enough, $g^{n}$ is $\left(r, \varepsilon_{n}\right)$-proximal with $\varepsilon_{n} \underset{n \rightarrow \infty}{\rightarrow} 0$.

Since GL $(V)$ is endowed with a Euclidean norm, it admits a canonical basis $\left(e_{j}\right)_{1 \leq j \leq \operatorname{dim}(V)}$. We set $x_{0}:=\mathbb{P}\left(e_{1}\right)$ and $H_{0}:=\mathbb{P}\left(\oplus_{j=2}^{\operatorname{dim}(V)} \mathbb{R} e_{j}\right)$. Recall that $\mathrm{GL}(V)$ admits a polar decomposition i.e. for any $g \in \mathrm{GL}(V)$, there exists orthogonal endomorphisms $k_{g}, l_{g} \in \mathrm{O}(V)$ and a unique symmetric endomorphism $a_{g}$ of eigenvalues $\left(a_{g}(j)\right)_{1 \leq j \leq \operatorname{dim}(V)}$ with $a_{g}(1) \geq a_{g}(2) \geq \ldots \geq a_{g}(\operatorname{dim}(V))$ such that $g=k_{g} a_{g} l_{g}$. Let us introduce a key [BG03, Lemma 3.4], due to Breuillard and Gelander, which is needed to obtain the Lipschitz properties.

Lemma 3.4 ([BG03]). Let $r, \delta \in(0,1]$. Let $g \in \mathrm{GL}(V)$. If $\left|\frac{a_{g}(2)}{a_{g}(1)}\right| \leq \delta$, then $g$ is $\frac{\delta}{r^{2}}-$ Lipschitz on $\mathcal{V}_{r}\left(l_{g}^{-1} H_{0}\right)^{c}$.

Proof of Lemma 3.3. Let $g \in \mathrm{GL}(V)$ be a proximal element. Set $r:=\frac{1}{2} d\left(x_{+}(g), X_{-}(g)\right)$. By proximality, $r$ is positive. Let us prove that for all $0<\varepsilon \leq r$, there exists $n_{0}$ such that $g^{n}$ is $(r, \varepsilon)$-proximal for all $n \geq n_{0}$.

Denote by $\pi_{g}$ the projector of kernel $V_{-}(g)$ and of image $V_{+}(g)$. Then

$$
\frac{g^{n}}{\lambda_{1}(g)^{n}}=\pi_{g}+\frac{g_{\mid V_{-}(g)}^{n}}{\lambda_{1}(g)^{n}} .
$$

By proximality, the highest eigenvalue of $g_{\mid V_{-}(g)}$ is strictly smaller than $\lambda_{1}(g)$. It follows immediately by the Spectral Radius Formula that $\frac{g^{n}}{\lambda_{1}(g)^{n}} \underset{n \rightarrow+\infty}{\longrightarrow} \pi_{g}$. Hence for any $y \in X \backslash X_{-}(g)$, uniformly on any compact subset of $X \backslash X_{-}(g)$,

$$
g^{n} \cdot y \underset{n \rightarrow+\infty}{\longrightarrow} x_{+}(g) \text {. }
$$

It remains to show the Lipschitz properties of $g^{n}$, for $n$ big enough. For all $n \in \mathbb{N}$, we denote by $k_{n}, l_{n}$ (resp. $a_{n}$ ) the orthogonal (resp. symmetric) components of $g^{n}$ so that $g_{n}=k_{n} a_{n} l_{n}$. We also set $x_{n}:=k_{n} x_{0}$ and $H_{n}:=l_{n}^{-1} H_{0}$.

For any $n \geq 1$, denote by $p_{x_{n}, H_{n}}$ the endomorphism of norm 1 such that $\mathbb{P}\left(\operatorname{im}\left(p_{x_{n}, H_{n}}\right)\right)=x_{n}$ and $\mathbb{P}\left(\operatorname{ker}\left(p_{x_{n}, H_{n}}\right)\right)=H_{n}$. Then by polar decomposition,

$$
\frac{g^{n}}{a_{n}(1)}=p_{x_{n}, H_{n}}+O\left(\frac{a_{n}(2)}{a_{n}(1)}\right) \text {. }
$$


By the Spectral Radius Formula, $\left|\frac{a_{n}(2)}{a_{n}(1)}\right|^{\frac{1}{n}} \underset{n \rightarrow \infty}{\longrightarrow} \frac{\lambda_{1}\left(g_{\mid V_{-}(g)}\right)}{\lambda_{1}(g)}<1$. Hence

$$
\lim _{n \rightarrow \infty} \frac{a_{n}(2)}{a_{n}(1)}=0
$$

Let $(x, H)$ be an accumulating point of the sequence $\left(x_{n}, Y_{n}\right)_{n \geq 1}$. Then there is a converging subsequence $x_{\varphi(n)}, Y_{\varphi(n)} \underset{n \rightarrow+\infty}{\longrightarrow} x, Y$. Denote by $p_{x, Y}$ the endomorphism of norm 1 such that $\mathbb{P}\left(\operatorname{im}\left(p_{x, Y}\right)\right)=x$ and $\mathbb{P}\left(\operatorname{ker}\left(p_{x, Y}\right)\right)=Y$. Then,

$$
\frac{g^{\varphi(n)}}{a_{\varphi(n)}(1)} \underset{n \rightarrow+\infty}{\longrightarrow} p_{x, Y} .
$$

It allows us to deduce in particular, that for any $y \in X \backslash\left\{H, X_{-}(g)\right\}$,

$$
g^{\varphi(n)} \cdot y \underset{n \rightarrow+\infty}{\longrightarrow} x \text {. }
$$

However, by proximality of $g$ and uniqueness of the limit, we obtain that $x=x_{+}(g)$.

Similarly, by duality, we obtain that $Y=X_{-}(g)$. Hence $\left(x_{n}, Y_{n}\right)_{n \geq 1}$ converges towards $\left(x_{+}(g), X_{-}(g)\right)$.

Fix $0<\varepsilon \leq r$. Then for $n$ large enough, the inclusion $\mathcal{V}_{\varepsilon}\left(X_{-}(g)\right) \supset \mathcal{V}_{\frac{\varepsilon}{2}}\left(H_{n}\right)$ holds. By Lemma 3.4, the restriction of $g^{n}$ to $\mathcal{V}_{\varepsilon}\left(X_{-}(g)\right)^{c} \subset \mathcal{V}_{\frac{\varepsilon}{2}}\left(H_{n}\right)^{c}$ is then a $\left|\frac{a_{n}(2)}{a_{n}(1)}\right| \frac{4}{\varepsilon^{2}}-$ Lipschitz map. Finally, for $n$ large enough so that $\left|\frac{a_{n}(2)}{a_{n}(1)}\right| \frac{4}{\varepsilon^{2}}<\varepsilon$, the restriction of $g^{n}$ to $\mathcal{V}_{\varepsilon}\left(X_{-}(g)\right)^{c}$ is $\varepsilon$-Lipschitz.

The following proximality criterion is due to Tits [Tit71] and one can find the statement under this form in [Ben00].

Lemma 3.5. Fix $0<\varepsilon \leq r$. Let $x \in \mathbb{P}(V)$ and a hyperplane $Y \subset \mathbb{P}(V)$ such that $d(x, Y) \geq 6 r$. Let $g \in \mathrm{GL}(V)$. If

(i) $g \mathcal{V}_{\varepsilon}(Y)^{c} \subset B(x, \varepsilon)$,

(ii) $g$ restricted to $\mathcal{V}_{\varepsilon}(Y)^{c}$ is $\varepsilon$-Lipschitz,

then $g$ is $(2 r, 2 \varepsilon)$-proximal. Furthermore, the attractive point $x_{+}(g)$ is in $B(x, \varepsilon)$ and the repulsive hyperplane $X_{-}(g)$ in a $\varepsilon-$ neighbourhood of $Y$.

Corollary 3.6. Fix $0<\varepsilon \leq r$. Let $g \in \mathrm{GL}(V)$ be $a(r, \varepsilon / 2)$-proximal element so that $d\left(x_{+}(g), X_{-}(g)\right) \geq 7 r$.

Then for any $h \in \mathrm{GL}(V)$ so that $\left\|h-i d_{V}\right\| \leq \varepsilon / 2$, the product gh is $(2 r, 2 \varepsilon)$-proximal, with $x_{+}(g h) \in B\left(x_{+}(g), \varepsilon\right)$.

Proof. Consider a $(r, \varepsilon / 2)$-proximal element $g$ and $h \in \mathrm{GL}(V)$ as in the hypothesis.

Remark that $g h$ maps $h^{-1} \mathcal{V}_{\varepsilon / 2}\left(X_{-}(g)\right)^{c}$ towards the open ball $B\left(x_{+}(g), \varepsilon / 2\right)$. Furthermore, by proximality of $g$, the restriction of $g h$ to $h^{-1} \mathcal{V}_{\varepsilon / 2}\left(X_{-}(g)\right)^{c}$ is $\varepsilon / 2$-Lipschitz.

Since $h$ is close to $i d_{V}$, then $\mathcal{V}_{\varepsilon}\left(h^{-1} X_{-}(g)\right)^{c} \subset h^{-1} \mathcal{V}_{\varepsilon / 2}\left(X_{-}(g)\right)^{c}$. Hence $g h$ restricted to $\mathcal{V}_{\varepsilon}\left(h^{-1} X_{-}(g)\right)^{c}$ is $\varepsilon-$ Lipschitz of image in the open ball $B\left(x_{+}(g), \varepsilon\right)$. Furthermore, $d\left(x_{+}(g), h^{-1} X_{-}(g)\right) \geq$ $d\left(x_{+}(g), X_{-}(g)\right)-\varepsilon>7 r-\varepsilon \geq 6 r$.

Finally, by Lemma 3.5 , we deduce that $g h$ is $(2 r, 2 \varepsilon)$-proximal, with $x_{+}(g h) \in B\left(x_{+}(g), \varepsilon\right)$.

For all proximal elements $g, h$ of $\operatorname{End}(V)$ such that $x_{+}(h) \notin X_{-}(g)$, we consider two unit eigenvectors $v_{+}(h) \in x_{+}(h)$ and $v_{+}(g) \in x_{+}(g)$ and denote by $\alpha(g, h)$ the unique real number such that $v_{+}(h)-\alpha(g, h) v_{+}(g) \in V_{-}(g)$. A priori, $\alpha(g, h)$ depends on the choice of the unit vectors. However, its absolute value does not. 
Given $g_{1}, \ldots g_{l}$ of $\operatorname{End}(V)$, set $g_{0}=g_{l}$ and assume $x_{+}\left(g_{i-1}\right) \notin X_{-}\left(g_{i}\right)$ for all $1 \leq i \leq l$. We set

$$
\nu_{1}\left(g_{l}, \ldots, g_{1}\right)=\sum_{1 \leq j \leq l} \log \left|\alpha\left(g_{j}, g_{j-1}\right)\right| .
$$

This product does not depends on the choices of unit eigenvectors for $g_{j}$.

The following proposition explains how to control the spectral radius $\lambda_{1}(\gamma)$ when $\gamma$ is a product of $(r, \epsilon)$-proximal elements.

Proposition 3.7 ([Ben00]). For all $0<\epsilon \leq r$, there exist positive constants $C_{r, \epsilon}$ such that for all $r>0, \lim _{\epsilon \rightarrow 0} C_{r, \epsilon}=0$ and such that the following holds. If $\gamma_{1}, \ldots \gamma_{l}$ are $(r, \varepsilon)$-proximal elements, such that $d\left(x_{+}\left(\gamma_{i-1}\right), X_{-}\left(\gamma_{i}\right)\right) \geq 6 r$ for all $1 \leq i \leq l$ with $\gamma_{0}=\gamma_{l}$, then for all $n_{1}, \ldots, n_{l} \geq 1$,

$$
\left|\log \left(\lambda_{1}\left(\gamma_{l}^{n_{l}} \ldots \gamma_{1}^{n_{1}}\right)\right)-\sum_{i=1}^{l} n_{i} \log \left(\lambda_{1}\left(\gamma_{i}\right)\right)-\nu_{1}\left(\gamma_{l}, \ldots, \gamma_{1}\right)\right| \leq l C_{r, \epsilon} .
$$

Furthermore, the map $\gamma_{l}^{n_{l}} \ldots \gamma_{1}^{n_{1}}$ is $(2 r, 2 \varepsilon)$-proximal with $x_{+}\left(\gamma_{l}^{n_{l}} \ldots \gamma_{1}^{n_{1}}\right) \in B\left(x_{+}\left(\gamma_{l}\right), \varepsilon\right)$ and $X_{-}\left(\gamma_{l}^{n_{l}} \ldots \gamma_{1}^{n_{1}}\right) \subset \mathcal{V}_{\varepsilon}\left(X_{-}\left(\gamma_{1}\right)\right)$.

Proof. Taking the logarithm in Benoist's [Ben00, Lemma 1.4] gives us the first part of the statement (the estimates). We only give a proof of the proximality and the localisation of the attractive points and repulsive hyperplane.

Let $n_{1}, \ldots, n_{l} \geq 1$ and assume that $0<\epsilon \leq r$ and $\epsilon<1$. Let us prove that $g_{n}:=\gamma_{l}^{n_{l}} \ldots \gamma_{1}^{n_{1}}$ verifies the assumptions (i) (ii) of the proximality criterion Lemma 3.5. More precisely, we prove by induction on $l$ that $g_{n}$ restricted to $\mathcal{V}_{\varepsilon}\left(X_{-}\left(\gamma_{1}\right)\right)^{c}$ is $\varepsilon-\operatorname{Lipschitz}$ and $g_{n} \mathcal{V}_{\varepsilon}\left(X_{-}\left(\gamma_{1}\right)\right)^{c} \subset$ $B\left(x_{+}\left(\gamma_{l}\right), \varepsilon\right)$.

By $(r, \varepsilon)$-proximality of $\gamma_{1}^{n_{1}}$, the restriction of $\gamma_{1}^{n_{1}}$ to $\mathcal{V}_{\varepsilon}\left(\gamma_{1}\right)^{c}$ is $\varepsilon-\operatorname{Lipschitz}$ and $\gamma_{1}^{n_{1}} \mathcal{V}_{\varepsilon}\left(\gamma_{1}\right)^{c} \subset$ $B\left(x_{+}\left(\gamma_{1}\right), \varepsilon\right)$.

Assume that for some $1 \leq i \leq l$ that $\gamma_{i}^{n_{i}} \ldots \gamma_{1}^{n_{1}}$ restricted to $\mathcal{V}_{\varepsilon}\left(X_{-}\left(\gamma_{1}\right)\right)^{c}$ is $\varepsilon$-Lipschitz and $\gamma_{i}^{n_{i}} \ldots \gamma_{1}^{n_{1}} \mathcal{V}_{\varepsilon}\left(X_{-}\left(\gamma_{1}\right)\right)^{c} \subset \bar{B}\left(x_{+}\left(\gamma_{i}\right), \varepsilon\right)$. Since $d\left(x_{+}\left(\gamma_{i}\right), X_{-}\left(\gamma_{i+1}\right)\right) \geq 6 r$ and $0<\epsilon \leq r$ we obtain $B\left(x_{+}\left(\gamma_{i}\right), \varepsilon\right) \subset \mathcal{V}_{\varepsilon}\left(X_{-}\left(\gamma_{i+1}\right)\right)^{c}$. Then using $(r, \varepsilon)$-proximality of $\gamma_{i+1}$, its restriction to $B\left(x_{+}\left(\gamma_{i}\right), \varepsilon\right)$ is $\varepsilon$-Lipschitz and $\gamma_{i+1}^{n_{i+1}} B\left(x_{+}\left(\gamma_{i}\right), \varepsilon\right) \subset B\left(x_{+}\left(\gamma_{i+1}\right), \varepsilon\right)$. Hence by induction hypothesis and using $\epsilon<1$, the map $\gamma_{i+1}^{n_{i+1}} \ldots \gamma_{1}^{n_{1}}$ restricted to $\mathcal{V}_{\varepsilon}\left(X_{-}\left(\gamma_{1}\right)\right)^{c}$ is $\varepsilon$-Lipschitz and $\gamma_{i+1}^{n_{i+1}} \ldots \gamma_{1}^{n_{1}} \mathcal{V}_{\varepsilon}\left(X_{-}\left(\gamma_{1}\right)\right)^{c} \subset B\left(x_{+}\left(\gamma_{i+1}\right), \varepsilon\right)$

We conclude the proof. By assumption, $d\left(x_{+}\left(\gamma_{l}\right), X_{-}\left(\gamma_{1}\right)\right) \geq 6 r$. Finally, by Lemma 3.5 we deduce $(2 r, 2 \varepsilon)$-proximality of $g_{n}$ with $x_{+}\left(g_{n}\right) \in B\left(x_{+}\left(\gamma_{l}\right), \varepsilon\right)$ and $X_{-}\left(g_{n}\right) \subset \mathcal{V}_{\varepsilon}\left(X_{-}\left(\gamma_{1}\right)\right)$.

The previous proposition motivates the next definition.

Definition 3.8. Let $0<\varepsilon \leq r$. A semigroup $\Gamma \subset \mathrm{GL}(V)$ is strongly $(r, \varepsilon)$-Schottky if

(i) every $h \in \Gamma$ is $(r, \varepsilon)$-proximal,

(ii) $d\left(x_{+}(h), X_{-}\left(h^{\prime}\right)\right) \geq 6 r$ for all $h, h^{\prime} \in \Gamma$.

We also write that $\Gamma$ is a strong $(r, \varepsilon)$-Schottky semigroup.

3.2. Representations of a semisimple Lie group $G$. Let $(V, \rho)$ be a representation of $G$ in a real vector space of finite dimension. For every character $\chi$ of $\mathfrak{a}$, denote the associated eigenspace by $V_{\chi}:=\{v \in V \mid \forall a \in \mathfrak{a}, \rho(a) v=\chi(a) v\}$. The set of restricted weights of $V$ is the set $\Sigma(\rho):=\left\{\chi \mid V_{\chi} \neq 0\right\}$. Simultaneous diagonalization leads to the decomposition $V=\underset{\chi \in \Sigma(\rho)}{\oplus} V_{\chi}$. The set of weights is partially ordered as follows

$$
\left(\chi_{1} \leq \chi_{2}\right) \Leftrightarrow\left(\forall a \in A^{+}, \quad \chi_{1}(a) \leq \chi_{2}(a)\right) .
$$


Whenever $\rho$ is irreducible, the set $\Sigma(\rho)$ has a highest element $\chi_{\max }$ which is the highest restricted weight of $V$. Denote by $V_{\rho}$ the eigenspace of the highest restricted weight, and by $Y_{\rho}$ the $\mathfrak{a}$-invariant supplementary subspace of $V_{\rho}$ i.e. $Y_{\rho}:=\operatorname{ker}\left(V_{\chi_{\max }}^{*}\right)=\underset{\chi \in \Sigma(\rho) \backslash\left\{\chi_{\max }\right\}}{\oplus} V_{\chi}$.

The irreducible representation $\rho$ is proximal when $\operatorname{dim}\left(V_{\chi_{\max }}\right)=1$. The following Lemma can be found in [BQ16, Lemma 5.32]. It is due to Tits [Tit71].

Denote by $\Pi \subset \Sigma^{+}$the subset of simple roots of the set of positive roots for the adjoint representation of $G$.

Lemma 3.9 ([Tit71]). For every simple root $\alpha \in \Pi$, there exists a proximal irreducible algebraic representation $\left(\rho_{\alpha}, V_{\alpha}\right)$ of $G$ whose highest weight $\chi_{\max , \alpha}$ is orthogonal to $\beta$ for every simple root $\beta \neq \alpha$.

These weights $\left(\chi_{\max , \alpha}\right)_{\alpha \in \Pi}$ form a basis of the dual space $\mathfrak{a}^{*}$.

Moreover, the map

$$
\begin{aligned}
& \mathcal{F}(X) \stackrel{y}{\longrightarrow} \prod_{\alpha \in \Pi} \mathbb{P}\left(V_{\alpha}\right) \\
\eta:=k_{\eta} \eta_{0} \longmapsto & \longmapsto
\end{aligned}
$$

is an embedding of the set of asymptotic Weyl chambers in this product of projective spaces.

We also define a dual map $H: \mathcal{F}(X) \rightarrow \prod_{\alpha \in \Pi} G r_{\operatorname{dim}\left(V_{\alpha}\right)-1}\left(V_{\alpha}\right)$ as follows. For every $\eta \in$ $\mathcal{F}(X)$, let $k_{\eta} \in K$ be an element so that $\eta=k_{\eta} \check{\eta}_{0}$ then

$$
\begin{aligned}
& \mathcal{F}(X) \stackrel{Y}{\longrightarrow} \prod_{\alpha \in \Pi} G r_{\operatorname{dim}\left(V_{\alpha}\right)-1}\left(V_{\alpha}\right) \\
\xi:=k_{\xi} \check{\eta}_{0} \longmapsto & \left(Y_{\alpha}(\xi):=\rho_{\alpha}\left(k_{\xi}\right) Y_{\rho_{\alpha}}\right)_{\alpha \in \Pi} .
\end{aligned}
$$

The maps $y$ and $Y$ provide us two ways to embed the space of asymptotic Weyl chambers $\mathcal{F}(X)$.

Corollary 3.10. The map

$$
\begin{aligned}
\mathcal{F}^{(2)}(X) \longrightarrow \prod_{\alpha \in \Pi} \mathbb{P}\left(V_{\alpha}\right) \oplus G r_{\operatorname{dim}(V)-1}\left(V_{\alpha}\right) \\
\left(f_{+}, f_{-}\right) \longmapsto\left(y_{\alpha}\left(f_{+}\right) \oplus Y_{\alpha}\left(f_{-}\right)\right)_{\alpha \in \Pi} .
\end{aligned}
$$

is a $G$-equivariant embedding of the space of flags in general position into this product of projective spaces in general position i.e. the associated subspaces are in direct sum.

Now we give an interpretation of the Cartan projection, the Iwasawa cocycle and the Jordan projection in terms of representations of $G$. The complete proof can be found in [BQ16].

Lemma 3.11 (Lemma 5.33 [BQ16]). Let $\alpha \in \Pi$ be a simple root and consider $\left(V_{\alpha}, \rho_{\alpha}\right)$ the proximal representation of $G$ given by Lemma 3.9. Then

(a) there exists a $\rho_{\alpha}(K)$-invariant Euclidean norm on $V_{\alpha}$ such that, for all $a \in A$, the endomorphism $\rho_{\alpha}(a)$ is symmetric.

(b) for such a norm and the corresponding subordinate norm on $\operatorname{End}\left(V_{\alpha}\right)$, for all $g \in G$, $\eta \in \mathcal{F}(X)$ and $v_{\eta} \in y_{\alpha}(\eta)$, one has

(i) $\chi_{\max , \alpha}(\mu(g))=\log \left(\left\|\rho_{\alpha}(g)\right\|\right)$,

(ii) $\chi_{\max , \alpha}(\lambda(g))=\log \left(\lambda_{1}\left(\rho_{\alpha}(g)\right)\right)$,

(iii) $\chi_{\max , \alpha}(\sigma(g, \eta))=\log \frac{\left\|\rho_{\alpha}(g) v_{\eta}\right\|}{\left\|v_{\eta}\right\|}$.

The following lemma gives estimations on the Cartan projection of products of any pair of elements in $G$. 
Lemma 3.12. There exists a continuous, left and right $K$-invariant, function $h \in G \mapsto C_{h} \in$ $\mathbb{R}_{+}$such that

(i) for any $g \in G$, the Cartan projections $\mu(g h)-\mu(g)$ and $\mu(h g)-\mu(g)$ are in the ball $\overline{B_{\mathfrak{a}}\left(0, C_{h}\right)}$,

(ii) for any $\eta \in \mathcal{F}(X)$, the Iwasawa cocycle $\sigma(h, \eta) \in \overline{B_{\mathfrak{a}}\left(0, C_{h}\right)}$.

Proof. Abusing terminology, we say that a function is $K$-invariant when it is $K$-invariant for both left and right action.

Let us prove the first point. For any $\alpha \in \Pi$, we consider the proximal irreducible representation $\left(\rho_{\alpha}, V_{\alpha}\right)$ of $G$ given by Lemma 3.9 .

Using Lemma 3.11, we endow each vector space $V_{\alpha}$ with $\rho_{\alpha}(K)$-invariant Euclidean norm. Classical properties of the norm lead, for all $\alpha \in \Pi$ and every $g, h \in G$, to

$$
\begin{aligned}
& \frac{\left\|\rho_{\alpha}(g)\right\|}{\left\|\rho_{\alpha}\left(h^{-1}\right)\right\|} \leq\left\|\rho_{\alpha}(g h)\right\| \leq\left\|\rho_{\alpha}(g)\right\|\left\|\rho_{\alpha}(h)\right\|, \\
& \frac{1}{\left\|\rho_{\alpha}\left(h^{-1}\right)\right\|} \leq \frac{\left\|\rho_{\alpha}(g h)\right\|}{\left\|\rho_{\alpha}(g)\right\|} \leq\left\|\rho_{\alpha}(h)\right\| .
\end{aligned}
$$

Note that we obtain the same inequalities for $h g$. s By Lemma 3.11, we deduce

$$
-\chi_{\max , \alpha}\left(\mu\left(h^{-1}\right)\right) \leq \chi_{\max , \alpha}(\mu(g h)-\mu(g)) \leq \chi_{\max , \alpha}(\mu(h)) .
$$

For any $\alpha \in \Pi$, set $h_{\alpha}:=\max \left(\chi_{\max , \alpha}(\mu(h)), \chi_{\max , \alpha}\left(\mu\left(h^{-1}\right)\right)\right)$. Furthermore, by Lemma 3.9, the weights $\left(\chi_{\max , \alpha}\right)_{\alpha \in \Pi}$ form a basis of the dual space $\mathfrak{a}^{*}$. In other word, they admit a dual basis in $\mathfrak{a}$. Denote by $C_{h}>0$ the real number such that $\overline{B_{\mathfrak{a}}\left(0, C_{h}\right)}$ is the smallest closed ball containing any point of dual coordinates in $\left(\left[-h_{\alpha}, h_{\alpha}\right]\right)_{\alpha \in \Pi}$ for the dual basis of $\left(\chi_{\max , \alpha}\right)_{\alpha \in \Pi \text {. }}$ Hence $\overline{B_{\mathfrak{a}}\left(0, C_{h}\right)}$ is compact and contains $\mu(g h)-\mu(g)$ and $\mu(h g)-\mu(g)$.

It remains to show that the function $h \mapsto C_{h}$ is continuous and $K$-invariant. It is due to the fact that the Cartan projection and the map $h \mapsto \mu\left(h^{-1}\right)$ are both continuous and $K$-invariant. Hence, by taking the supremum in each coordinate, the map $h \mapsto\left(h_{\alpha}\right)_{\alpha \in \Pi}$ is continuous and $K$-invariant. Furthermore, by definition of $C_{h}$, we obtain $K$-invariance and continuity of $h \mapsto C_{h}$.

Similarly, the second point is a direct consequence of Lemma 3.11, (i) and (iii) and of the inequality

$$
\frac{1}{\left\|\rho_{\alpha}\left(h^{-1}\right)\right\|} \leq \frac{\left\|\rho_{\alpha}(h)\left(v_{\eta}\right)\right\|}{\left\|v_{\eta}\right\|} \leq\left\|\rho_{\alpha}(h)\right\|
$$

where $\eta \in \mathcal{F}(X)$ and $v_{\eta} \in V_{\alpha}$ is the associated non trivial vector.

3.3. Loxodromic elements. Let us now study the dynamical properties of the loxodromic elements in the representations of the previous paragraph. [BQ16, Lemma 5.37] states that any element of $G$ is loxodromic if and only if its image is proximal for every representations given by Lemma 3.9. This allows to extend the notions and results on proximal elements to loxodromic elements in $G$.

Definition 3.13. An element $g \in G$ is loxodromic if its Jordan projection $\lambda(g)$ is in the interior of the Weyl chamber $\mathfrak{a}^{++}$or (equivalently) if for all $\alpha \in \Pi$ the endomorphisms $\rho_{\alpha}(g)$ are proximal.

Let $0<\varepsilon \leq r$. An element $g \in G$ is $(r, \varepsilon)$-loxodromic if for all $\alpha \in \Pi$ the endomorphisms $\rho_{\alpha}(g)$ are $(r, \varepsilon)$-proximal.

A semigroup $\Gamma$ of $G$ is strongly $(r, \varepsilon)$-Schottky if for all $\alpha \in \Pi$ the semigroups $\rho_{\alpha}(\Gamma) \subset$ $\operatorname{End}\left(V_{\alpha}\right)$ are strongly $(r, \varepsilon)$-Schottky. 
Attractive and repulsive asymptotic Weyl chambers of loxodromic elements were defined in section 2.2 as follows. For any loxodromic element $g \in G$, then $\left(g^{+}, g^{-}\right):=h_{g}\left(\eta_{0}, \check{\eta}_{0}\right) \in \mathcal{F}^{(2)}(X)$ where $h_{g} \in G$ is an element so that there is an angular part $m(g) \in M$ with $g=h_{g} e^{\lambda(g)} m(g) h_{g}^{-1}$.

The $G$-equivariant map $\left(f_{+}, f_{-}\right) \in \mathcal{F}^{(2)}(X) \rightarrow\left(y_{\alpha}\left(f_{+}\right) \oplus Y_{\alpha}\left(g_{-}\right)\right)_{\alpha \in \Pi}$ given by Corollary 3.10 allows to caracterize attractive points and repulsive points in $\mathcal{F}(X)$ for loxodromic elements.

Lemma 3.14. For any loxodromic element $g \in G$, the following statements are true.

(i) $g^{-1}$ is loxodromic, of attractive point $g^{-}$and repulsive point $g^{+}$,

(ii) the image of $\left(g^{+}, g^{-}\right) \in \mathcal{F}^{(2)}(X)$ by the above map is the family of attractive points and repulsive hyperplanes in general position $\left(x_{+}\left(\rho_{\alpha}(g)\right) \oplus X_{-}\left(\rho_{\alpha}(g)\right)\right)_{\alpha \in \Pi}$,

(iii) $g$ contracts any point $\eta \in \mathcal{F}(X)$ in general position with $g^{-}$, to $g^{+}$i.e. $\lim _{n \rightarrow+\infty} g^{n} \eta=g^{+}$,

(iv) for any nonempty open set $O_{-} \subset \mathcal{F}(X)$ in general position with $g^{+}$, for any nonempty open neighbourhood $U_{-} \subset \mathcal{F}(X)$ of $g^{-}$, there exists $N \in \mathbb{N}$ so that for any $n \geq N$, then $O_{-} \cap g^{n} U_{-} \neq \emptyset$.

Proof. Let $g \in G$ be a loxodromic element, consider an element $h_{g} \in G$ and an angular part $m(g) \in M$ so that $g=h_{g} m(g) e^{\lambda(g)} h_{g}^{-1}$. Then $g^{-1}=h_{g} m(g)^{-1} e^{-\lambda(g)} h_{g}^{-1}$. Remark that $-\lambda(g)$ is in the interior of the Weyl chamber $-\mathfrak{a}^{+}$. Consider the element of the Weyl group $N_{K}(A) / M$ whose adjoint action on $\mathfrak{a}$ sends $\mathfrak{a}^{+}$onto $-\mathfrak{a}^{+}$. Denote one representative by $k_{\iota} \in N_{K}(A)$. Then $-\operatorname{Ad}\left(k_{\iota}\right)(\lambda(g)) \in \mathfrak{a}^{++}$, hence

$$
g^{-1}=h_{g} k_{\iota}\left(k_{\iota}^{-1} m(g) k_{\iota}\right)^{-1} e^{-A d\left(k_{\iota}\right)(\lambda(g))}\left(h_{g} k_{\iota}\right)^{-1} .
$$

Next, we remark that $k_{\iota}^{-1} M k_{\iota}$ is in the centralizer of $k_{\iota}^{-1} A k_{\iota}=A$, hence $k_{\iota}^{-1} m(g) k_{\iota} \in k_{\iota}^{-1} M k_{\iota}=$ $M$. We deduce that $\lambda\left(g^{-1}\right)=-A d\left(k_{\iota}\right)(\lambda(g))$ and set $h_{g^{-1}}=h_{g} k_{\iota}$ with the angular part $m\left(g^{-1}\right)=\left(k_{\iota}^{-1} m(g) k_{\iota}\right)^{-1}$. Then the pair of attractive and repulsive points of $g^{-1}$ in $\mathcal{F}(X)$ is $\left(h_{g} k_{\iota} \eta_{0}, h_{g} k_{\iota} \check{\eta}_{0}\right)$. Since $k_{\iota} \eta_{0}=\check{\eta}_{0}$ and $k_{\iota} \check{\eta}_{0}=\eta_{0}$ we obtain the first statement i.e. that $g^{-}$ (resp. $g^{+}$) is the attractive (resp. repulsive) point of $g^{-1}$.

For the second point, it suffices to prove that for any loxodromic element $g \in G$, for every $\alpha \in \Pi$, the vector space $\rho_{\alpha}\left(h_{g}\right) V_{\rho_{\alpha}}=y_{\alpha}\left(g^{+}\right)$is the eigenspace associated to the spectral radius of $\rho_{\alpha}(g)$ and that $\rho_{\alpha}\left(h_{g}\right) Y_{\rho_{\alpha}}=Y_{\alpha}\left(g^{-}\right)$is the direct sum of the other eigenspaces.

Let $g \in G$ be a loxodromic element and let $\alpha \in \Pi$. By Lemma 3.11, the spectral radius of $\rho_{\alpha}(g)$ is $\exp \left(\chi_{\max , \alpha}(\lambda(g))\right)$. We deduce that the eigenspace of the highest eigenvalue is $\rho_{\alpha}\left(h_{g}\right) V_{\rho_{\alpha}}$. Furthermore, by definition of proximality, $x_{+}\left(\rho_{\alpha}(g)\right)=\mathbb{P}\left(\rho_{\alpha}\left(h_{g}\right) V_{\rho_{\alpha}}\right)=y_{\alpha}\left(g^{+}\right)$.

Remark that the other eigenvalues of $\rho_{\alpha}(g)$ are given by the other non maximal restricted weights of the representation $\left(\rho_{\alpha}, V_{\alpha}\right)$. Hence $\rho_{\alpha}\left(h_{g}\right) Y_{\rho_{\alpha}}$ is the direct sum of the other eigenspaces of $\rho_{\alpha}\left(h_{g}\right)$. The projective space $\mathbb{P}\left(\rho_{\alpha}\left(h_{g}\right) Y_{\rho_{\alpha}}\right)$ is thus the repulsive hyperplane of $\rho_{\alpha}(g)$. Hence the second statement is true.

For any point $\eta \in \mathcal{F}(X)$ in general position with $g^{-}$and for any $\alpha \in \Pi$, the point $y_{\alpha}(\eta)$ is then in general position with the hyperplane $Y_{\alpha}\left(g^{-}\right)$. Hence $\lim _{n \rightarrow+\infty} \rho_{\alpha}\left(g^{n}\right) y_{\alpha}(\eta)=x_{+}\left(\rho_{\alpha}(g)\right)$. This gives the third statement.

For the last statement, we apply the third statement to $g^{-1}$. It means that, for any nonempty open set $O_{-} \subset \mathcal{F}(X)$ in general position with $g^{+}$and for any nonempty open neighbourhood $U_{-} \subset \mathcal{F}(X)$ of $g^{-}$, there exists $N \in \mathbb{N}$ so that for any $n \geq N$, then

$$
\left(g^{-1}\right)^{n} O_{-} \cap U_{-} \neq \emptyset \text {. }
$$

Hence, for any $n \geq N$,

finally,

$$
g^{n}\left(g^{-n} O_{-} \cap U_{-}\right) \neq \emptyset,
$$

$$
O_{-} \cap g^{n} U_{-} \neq \emptyset .
$$


Lemma 3.3 and Corollary 3.6 extend to loxodromic elements.

Lemma 3.15. For every loxodromic element $g \in G$, there exists $r>0$ and $n_{0} \in \mathbb{N}$ such that for all $n \geq n_{0}$ large enough, $g^{n}$ is $\left(r, \varepsilon_{n}\right)$-loxodromic with $\varepsilon_{n} \underset{n \rightarrow \infty}{\rightarrow} 0$.

Corollary 3.16. Fix $0<\varepsilon \leq r$. Let $g \in G$ be a $(r, \varepsilon / 2)$-loxodromic element so that $d\left(g^{+}, g^{-}\right) \geq$ $7 r$.

Then for any $h \in G$ so that $\left\|h-i d_{V}\right\| \leq \varepsilon / 2$, the product gh is $(2 r, 2 \varepsilon)$-loxodromic, with $(g h)^{+} \in B\left(g^{+}, \varepsilon\right)$.

Likewise, we generalize estimates of Proposition 3.7 to products of loxodromic elements of $G$ in general configuration.

Given $l$ loxodromic elements $g_{1}, \ldots g_{l}$ of $G$, set $g_{0}=g_{l}$ and assume that the asymptotic points $g_{i-1}^{+}$and $g_{i}^{-}$are opposite for all $1 \leq i \leq l$. Thanks to lemma 3.9, there exists a unique element $\nu=\nu\left(g_{1}, \ldots, g_{l}\right) \in \mathfrak{a}$ whose coordinates in the dual basis of $\left(\chi_{\alpha, \max }\right)_{\alpha \in \Pi}$ are

$$
\left(\chi_{\alpha, \max }(\nu)\right)_{\alpha \in \Pi}:=\left(\nu_{1}\left(\rho_{\alpha}\left(g_{1}\right), \ldots, \rho_{\alpha}\left(g_{l}\right)\right)\right)_{\alpha \in \Pi} .
$$

This product does not depends on the choices of unit eigenvectors for $g_{j}$. The product of projective spaces $\prod_{\alpha \in \Pi} \mathbb{P}\left(V_{\alpha}\right)$ is endowed with the natural distance.

Proposition 3.17 (Benoist[Ben00]). For all $0<\epsilon \leq r$, there exist positive constants $C_{r, \epsilon}$ such that for all $r>0, \lim _{\epsilon \rightarrow 0} C_{r, \epsilon}=0$ and such that the following holds. If $\gamma_{1}, \ldots \gamma_{l}$ are $(r, \varepsilon)$ loxodromic elements, such that for all $1 \leq i \leq l$ with $\gamma_{0}=\gamma_{l}$ we have $d\left(y\left(\gamma_{i-1}^{+}\right), H\left(\gamma_{i}^{-}\right)\right) \geq 6 r$, then for all $n_{1}, \ldots, n_{l} \geq 1$

$$
\lambda\left(\gamma_{l}^{n_{l}} \ldots \gamma_{1}^{n_{1}}\right)-\sum_{i=1}^{l} n_{i} \lambda\left(\gamma_{i}\right)-\nu\left(\gamma_{l}, \ldots, \gamma_{1}\right) \in B_{\mathfrak{a}}\left(0, l C_{r, \epsilon}\right) .
$$

Furthermore, the map $g:=\gamma_{l}^{n_{l}} \ldots \gamma_{1}^{n_{1}}$ is $(2 r, 2 \varepsilon)$-loxodromic with $y\left(g^{+}\right) \in B\left(y\left(\gamma_{l}^{+}\right), \varepsilon\right)$ and $H\left(g^{-}\right) \in \mathcal{V}_{\varepsilon}\left(H\left(\gamma_{1}^{-}\right)\right)$

Using Proposition 3.17, one can construct finitely generated, strong $(r, \varepsilon)$-Schottky semigroups as follows. Let $0<\varepsilon \leq r$.

Let $S \subset G$ be a family of $(r / 2, \varepsilon / 2)$-loxodromic elements such that $d\left(y\left(h^{+}\right), H\left(h^{\prime-}\right)\right) \geq 7 r$ for all $h, h^{\prime} \in S$. Denote by $\Gamma^{\prime}$ the semigroup generated by $S$. Then every element $g \in \Gamma$ is a noncommuting product of proximal elements of the form $g_{l}^{n_{l}} \ldots g_{1}^{n_{1}}$ with $n_{1}, \ldots, n_{l} \geq 1$ and $g_{i} \neq$ $g_{i+1} \in S$ for all $1 \leq i<l$. By Proposition 3.17, we deduce $d\left(y\left(g^{+}\right), H\left(g^{-}\right)\right) \geq d\left(y\left(g_{l}^{+}\right), H\left(g_{1}^{-}\right)\right)-$ $\varepsilon \geq 6 r$ and that $g$ is $(r, \varepsilon)$-loxodromic. Thus, $\Gamma^{\prime}$ is strongly $(r, \varepsilon)$-Schottky.

\section{TOPOLOGICAL TRANSITIVITY}

Recall the definition of topological transitivity. We denote by $\mathfrak{a}_{1}^{+}$(resp. $\mathfrak{a}_{1}^{++}$) the intersection of the unit sphere in $\mathfrak{a}$ with $\mathfrak{a}^{+}\left(\right.$resp. $\left.\mathfrak{a}^{++}\right)$.

Definition 4.1. Let $\widetilde{\Omega} \subset \mathcal{W}(X)$ a $\Gamma$-invariant and $\mathfrak{a}$-invariant subset of parametric flats $\mathcal{W}(X)$. Let $\Omega:=\Gamma \backslash \widetilde{\Omega}$. Fix a direction $\theta \in \mathfrak{a}_{1}^{++}$. The Weyl chamber flow $\phi_{\mathbb{R}}^{\theta}$ is topologically transitive on $\Omega$ if for all open nonempty subsets $U, V \subset \Omega$, there exists $t_{n} \rightarrow+\infty$ such that for every $n \geq 1$, we have $U \cap \phi_{t_{n}}^{\theta}(V) \neq \emptyset$.

It is a standard fact that it is equivalent to one the following properties :

(1) there is a $\phi_{\mathbb{R}}^{\theta}$-dense orbit in $\Omega$. 
(2) for all open nonempty subsets $\widetilde{U}, \widetilde{V} \subset \widetilde{\Omega}(X)$, there exists $t_{n} \rightarrow+\infty$ such that for every $n \geq 1, \Gamma \widetilde{U} \cap \phi_{t_{n}}^{\theta}(\widetilde{V}) \neq \emptyset$.

(3) for all open nonempty subsets $\widetilde{U}, \widetilde{V} \subset \widetilde{\Omega}$, there exists $t_{n} \rightarrow+\infty$ such that for every $n \geq 1$, there exists $\gamma_{n} \in \Gamma$ with $\gamma_{n} \widetilde{U} \cap \phi_{t_{n}}^{\theta}(\widetilde{V}) \neq \emptyset$.

The equivalence between the definition and property (1) can be found in [Ebe72, Proposition 3.5]. The others equivalences are straightforward.

\subsection{Limit set, limit cone of Zariski dense subgroup.}

In the remaining parts of this paper, $\Gamma \subset G$ is a Zariski dense
semigroup of $G$.

Definition 4.2. A point $\eta \in \mathcal{F}(X)$ is a limit point if there exists a sequence $\left(\gamma_{n}\right)_{n \geq 1}$ in $\Gamma$ such that $\left(\gamma_{n}\left[f_{0}\left(a^{+}\right)\right]\right)_{n>1}$ converges in $\mathcal{F}(X)$ towards $\eta$.

The limit set of $\Gamma$, denoted by $L_{+}(\Gamma)$, is the set of limit points of $\Gamma$. It is a closed subset of $\mathcal{F}(X)$

Denote by $L_{-}(\Gamma)$ the limit set of $\Gamma^{-1}$ and finally let $L^{(2)}(\Gamma)=\left(L_{+}(\Gamma) \times L_{-}(\Gamma)\right) \cap \mathcal{F}^{(2)}(X)$ the subset of $\mathcal{F}^{2}(X)$ in general position.

Lemma 4.3 ([Ben97] Lemma 3.6 ). The set of pairs of attractive and repulsive points of loxodromic elements of $\Gamma$ is dense in $L_{+}(\Gamma) \times L_{-}(\Gamma)$.

Definition 4.4. We denote by $\widetilde{\Omega}(X)$ the subset of non-wandering Weyl chambers, defined through the Hopf parametrization by :

$$
\widetilde{\Omega}(X):=\mathcal{H}^{-1}\left(L^{(2)}(\Gamma) \times \mathfrak{a}\right) .
$$

This is a $\Gamma$-invariant subset of $\mathcal{W}(X)$. When $\Gamma$ is a subgroup, we denote by $\Omega(X):=\Gamma \backslash \widetilde{\Omega}(X)$ the quotient space.

Conze and Guivarc'h proved in [CG02, Theorem 6.4], the existence of dense $\mathfrak{a}-$ orbits in $\widetilde{\Omega}(X)$. By duality, it is equivalent to topological transitivity of left $\Gamma$-action on $\widetilde{\Omega}(X) / A M \simeq L^{(2)}(\Gamma)$. We propose a new simpler proof of this result adapting the one for negatively curved manifolds of Eberlein [Ebe72].

Theorem 4.5 ([CG02]). For any open nonempty subsets $\mathcal{U}^{(2)}, \mathcal{V}^{(2)} \subset L^{(2)}(\Gamma)$ there exists $g \in \Gamma$ such that $g \mathcal{U}^{(2)} \cap \mathcal{V}^{(2)} \neq \emptyset$.

Proof. Whithout loss of generality, we assume that $\mathcal{U}^{(2)}=\mathcal{U}_{+} \times \mathcal{U}_{-}$and $\mathcal{V}^{(2)}=\mathcal{V}_{+} \times \mathcal{V}_{-}$where $\mathcal{U}_{+}, \mathcal{V}_{+}\left(\right.$resp. $\left.\mathcal{U}_{-}, \mathcal{V}_{-}\right)$are open nonempty subsets of $L_{+}(\Gamma)\left(\right.$ resp. $\left.L_{-}(\Gamma)\right)$.

We choose an open set $W^{(2)}=W_{+} \times W_{-} \subset L^{(2)}(\Gamma)$ so that $\mathcal{V}_{+}$and $W_{-}$(resp. $W_{+}$and $\left.\mathcal{U}_{-}\right)$are opposite. Such a choice is always possible. If $\mathcal{V}_{+}$and $\mathcal{U}_{-}$are opposite, we can take $W^{(2)}=\mathcal{V}^{(2)}$. Otherwise, by taking $\mathcal{U}^{(2)}$ and $\mathcal{V}^{(2)}$ smaller, we can always assume that the subset of points in $L_{+}(\Gamma)\left(\right.$ resp. $\left.L_{-}(\Gamma)\right)$ in general position with $\mathcal{U}_{-}$(resp. $\left.\mathcal{V}_{+}\right)$is non empty. Then we choose a suitable opposite pair of open nonempty subsets $W_{+} \times W_{-} \subset L_{+}(\Gamma) \times L_{-}(\Gamma)$.

Since $W_{+} \times \mathcal{U}_{-} \subset L^{(2)}(\Gamma)$, then, by Lemma 4.3 , there are loxodromic elements in $\Gamma$ with attractive point in $W_{+}$and repulsive point in $\mathcal{U}_{-}$. By Lemma 3.14, such a loxodromic element $\gamma_{1}$ contracts points that are in general position with $\gamma_{1}^{-} \in \mathcal{U}_{-}$towards $\gamma_{1}^{+} \in W_{+}$. Apply now statement (iv) of Lemma 3.14, to loxodromic element $\gamma_{1}$, with $W_{-}$in general position with $\gamma_{1}^{+}$ and $U_{-}$containing $\gamma_{1}^{-}$. Hence for any $n$ large enough, $\gamma_{1}^{n} \mathcal{U}^{(2)} \cap W^{(2)} \neq \emptyset$.

We take an open subset $\mathcal{W}^{(2)}$ of $\gamma_{1}^{n} \mathcal{U}^{(2)} \cap W^{(2)}$ of the form $\mathcal{W}^{(2)}=\mathcal{W}_{+} \times \mathcal{W}_{-}$. Then $\mathcal{V}_{+} \times \mathcal{W}_{-} \subset$ $\mathcal{V}_{+} \times W_{-} \subset L^{(2)}(\Gamma)$. Likewise, we choose a loxodromic element $\gamma_{2} \in \Gamma$ so that $\gamma_{2} \mathcal{W}^{(2)} \cap \mathcal{V}^{(2)} \neq \emptyset$. 
Then

$$
\left(\gamma_{2} \gamma_{1}^{n} \mathcal{U}^{(2)} \cap \gamma_{2} W^{(2)}\right) \cap \mathcal{V}^{(2)} \supset \gamma_{2} \mathcal{W}^{(2)} \cap \mathcal{V}^{(2)} \neq \emptyset
$$

Finally, the element $g=\gamma_{2} \gamma_{1}^{n}$ satisfies $g \mathcal{U}^{(2)} \cap \mathcal{V}^{(2)} \neq \emptyset$

The theorem below describes the set of directions $\theta \in \mathfrak{a}_{1}^{+}$for which we will show that $\phi_{t}^{\theta}$ is topologically mixing.

Theorem 4.6 ([Ben97]). We define the limit cone of $\Gamma$ by, $\mathcal{C}(\Gamma):=\bigcup_{\gamma \in \Gamma} \mathbb{R} \lambda(\gamma)$. We have

$$
\mathcal{C}(\Gamma)=\bigcap_{n \geq 1} \bigcup_{\substack{\|\gamma\| \geq n \\ \gamma \in \Gamma}} \mathbb{R} \mu(\gamma) .
$$

Moreover this set is closed, convex, of non-empty interior.

4.2. Topological transitivity properties. Recall the definition of the subset of non-wandering Weyl chambers $\widetilde{\Omega}(X)=\mathcal{H}^{-1}\left(L^{(2)}(\Gamma) \times \mathfrak{a}\right)$.

Proposition 4.7. Let $\theta \in \mathfrak{a}^{++}$. If the flow $\left(\Omega(X), \phi_{\theta}^{t}\right)$ is topologically transitive then $\theta \in \stackrel{\circ}{\mathcal{C}}(\Gamma)$.

Proof. We assume that the dynamical system $\left(\Omega(X), \phi_{\theta}^{t}\right)$ is topologically transitive i.e. there exists a dense orbit. Let $x \in \Omega(X)$ be a point of $\phi_{\theta}^{t}$-dense orbit and choose $g_{x} \in G$ a lift of $x$ in $G$.

By density of $\left(\phi_{\theta}^{t}(x)\right)_{x \in \mathbb{R}}$, for any $y M \in \widetilde{\Omega}(X) \subset G / M$, there exists $t_{n} \rightarrow+\infty, \varepsilon_{n} \rightarrow i d_{G}$, $m_{n} \in M$ and $\gamma_{n} \in \Gamma$ so that

$$
\phi_{\theta}^{t_{n}}\left(g_{x}\right)=g_{x} e^{t_{n} \theta}=\gamma_{n} y \varepsilon_{n} m_{n} .
$$

In particular, since the element $y=g_{x} e^{-v}$ belongs to $\widetilde{\Omega}(X)$ for all $v \in \mathfrak{a}$, there exists $t_{n} \in$ $\mathbb{R}, \epsilon_{n} \rightarrow \operatorname{Id}_{G}, m_{n} \in M$ and $\gamma_{n} \in \Gamma$ such that

$$
g_{x} e^{t_{n} \theta}=\gamma_{n} g_{x} e^{-v} \varepsilon_{n} m_{n} .
$$

For every $n \geq 1$ we set $\varepsilon_{n}^{\prime}:=g_{x} e^{-v} \varepsilon_{n} e^{v} g_{x}^{-1}$. The sequence $\left(\varepsilon_{n}^{\prime}\right)_{n \geq 1}$ converges towards $i d_{G}$, and we have:

$$
g_{x} e^{v+t_{n} \theta} m_{n}^{-1} g_{x}^{-1}=\gamma_{n} \varepsilon_{n}^{\prime} .
$$

For every $n \geq 1$ we set $g_{n}:=g_{x} e^{v+t_{n} \theta} m_{n}^{-1} g_{x}^{-1}$.

Thanks to Lemma 3.12 , we deduce the following estimates

$$
\begin{aligned}
\mu\left(g_{n}\right) & \in v+t_{n} \theta+C_{g_{x}}+C_{g_{x}^{-1}} \\
\mu\left(\gamma_{n} \varepsilon_{n}^{\prime}\right) & \in \mu\left(\gamma_{n}\right)+C_{\varepsilon_{n}^{\prime}} .
\end{aligned}
$$

Therefore, $\mu\left(\gamma_{n}\right)$ is at bounded distance to $v+t_{n} \theta$ and by Theorem 4.6, $\theta$ must lie in the (closed) limit cone. We now show that $\theta$ cannot be in its boundary. For this we need to study more carefully the Jordan projection of $g_{n} \epsilon_{n}^{-1}$.

By definition, $\lambda\left(\gamma_{n}\right)$ belongs to the limit cone. By computing $\lambda\left(g_{n} \epsilon_{n}^{\prime-1}\right)$, we will show that $\lambda\left(\gamma_{n}\right)$ also lies in a uniform (with respect to $v$ ) neighborhood of $v+t_{n} \theta$. Finally, choosing $v$ far enough will force $\theta$ to be in the interior of the limit cone.

First of all, let us show that $g_{n} \epsilon_{n}^{\prime-1}$ is a loxodromic element. Since by hypothesis $\theta$ is in the interior of the Weyl chamber $\mathfrak{a}^{+}$, there exists $n_{0} \in \mathbb{N}$ so that for $n \geq n_{0}$ large enough, 
$\lambda\left(g_{n}\right)=v+t_{n} \theta \in \mathfrak{a}^{++}$. Hence $g_{n}$ is loxodromic and $\left(g_{n}^{+}, g_{n}^{-}\right)=\left(g_{x} \eta_{0}, g_{x} \check{\eta}_{0}\right)$ for all $n \geq n_{0}$. We choose $0<r \leq \frac{1}{7} d\left(g_{x} \eta_{0}, g_{x} \check{\eta}_{0}\right)$.

We apply Lemma 3.15 on the loxodromic elements $\left(g_{x} e^{\theta} g_{x}^{-1}\right)^{k}$. There is a sequence of $\rho_{k} \rightarrow$ 0 so that $\left(g_{x} e^{\theta} g_{x}^{-1}\right)^{k}$ is $\left(r, \rho_{k}\right)$-loxodromic. Then for any $n \geq n_{0}, g_{n}$ is the product of a $\left(r, \rho_{k_{n}}\right)$-loxodromic element and a loxodromic element of the form $g_{x} e^{v_{n}} m_{n}^{-1} g_{x}^{-1}$, where $v_{n} \in \mathfrak{a}^{+}$ is bounded, and with $k_{n} \rightarrow+\infty$. Since $g_{x} e^{v_{n}} m_{n}^{-1} g_{x}^{-1}$ and $\left(g_{x} e^{\theta} g_{x}^{-1}\right)^{k}$ have the same attractive and repulsive point in $\mathcal{F}(X)$, we deduce that $g_{n}$ is $\left(r, \rho_{k_{n}}\right)$-loxodromic for $n \geq n_{0}$. Take now $\rho_{n}^{\prime}=\max \left(\rho_{n}, \frac{1}{2}\left\|\varepsilon_{n}^{\prime}-i d_{G}\right\|\right)$. Then there exists $n_{1}$ so that for $n \geq \max \left(n_{0}, n_{1}\right)$, then $0<\rho_{n}^{\prime} \leq r$, and $g_{n}$ is $\left(r, \rho_{n}^{\prime}\right)$-loxodromic. Corollary 3.16 shows that $g_{n} \varepsilon_{n}^{\prime-1}$ is $\left(2 r, 2 \rho_{n}^{\prime}\right)$-loxodromic for $n$ large enough, and $\left(g_{n} \varepsilon_{n}^{\prime-1}\right)^{+} \in B\left(g_{x} \eta_{0}, \rho_{n}^{\prime}\right)$.

Using Fact 2.7, we compute $\lambda\left(g_{n} \varepsilon_{n}^{\prime-1}\right)$ :

$$
\begin{aligned}
& \lambda\left(g_{n} \varepsilon_{n}^{-1}\right)= \sigma\left(g_{n} \varepsilon_{n}^{\prime-1},\left(g_{n} \varepsilon_{n}^{\prime-1}\right)^{+}\right) \\
&=\sigma\left(g_{n}, \varepsilon_{n}^{\prime-1}\left(g_{n} \varepsilon_{n}^{\prime-1}\right)^{+}\right)+\sigma\left(\varepsilon_{n}^{\prime-1},\left(g_{n} \varepsilon_{n}^{\prime-1}\right)^{+}\right) \\
&=\sigma\left(g_{n}, g_{x} \eta_{0}\right) \\
& \quad+\left(\sigma\left(g_{n}, \varepsilon_{n}^{\prime-1}\left(g_{n} \varepsilon_{n}^{\prime-1}\right)^{+}\right)-\sigma\left(g_{n}, g_{x} \eta_{0}\right)\right) \\
& \quad+\sigma\left(\varepsilon_{n}^{\prime-1},\left(g_{n} \varepsilon_{n}^{\prime-1}\right)^{+}\right) .
\end{aligned}
$$

Remark that, $\sigma\left(g_{n}, g_{x} \eta_{0}\right)=\lambda\left(g_{n}\right)=v+t_{n} \theta$. hence

$$
\lambda\left(g_{n} \varepsilon_{n}^{\prime-1}\right)-\left(v+t_{n} \theta\right)=\left(\sigma\left(g_{n}, \varepsilon_{n}^{\prime-1}\left(g_{n} \varepsilon_{n}^{\prime-1}\right)^{+}\right)-\sigma\left(g_{n}, g_{x} \eta_{0}\right)\right)+\sigma\left(\varepsilon_{n}^{\prime-1},\left(g_{n} \varepsilon_{n}^{\prime-1}\right)^{+}\right) .
$$

We analyze separately the two terms of the right hand side of the last equality.

For the last term, by Lemma 3.12 (ii)

$$
\left\|\sigma\left(\varepsilon_{n}^{\prime-1},\left(g_{n} \varepsilon_{n}^{\prime-1}\right)^{+}\right)\right\| \leq C_{\varepsilon_{n}^{\prime}} .
$$

Now we will bound, independently of $v$, the term $\sigma\left(g_{n}, \varepsilon_{n}^{\prime-1}\left(g_{n} \varepsilon_{n}^{\prime-1}\right)^{+}\right)-\sigma\left(g_{n}, g_{x} \eta_{0}\right)$.

Let $\alpha \in \Pi$ be a simple root and consider the proximal representation of $G$ associated to $\alpha$. By Lemma 3.11 (b)(iii), for any $\xi \in \varepsilon_{n}^{\prime-1} B\left(g_{x} \eta_{0}, 2 \rho_{n}^{\prime}\right)$, there exists a non zero vector $v_{\xi} \in V_{\alpha}$ so that

$$
\chi_{\max , \alpha}\left(\sigma\left(g_{n}, \xi\right)\right)=\log \frac{\left\|\rho_{\alpha}\left(g_{n}\right) v_{\xi}\right\|}{\left\|v_{\xi}\right\|} .
$$

Let $\xi=\varepsilon_{n}^{\prime-1}\left(g_{n} \varepsilon_{n}^{\prime-1}\right)^{+}$and consider a unitary vector $v_{\xi} \in V_{\alpha}$. Since $\xi$ is in a $3 \rho_{n}^{\prime}-$ neighbourhood of $g_{n}^{+}$, we write $v_{\xi}=v_{+}+v_{<}$where $v_{+} \in V_{+}\left(\rho_{\alpha}\left(g_{n}\right)\right)$ and $v_{<} \in V_{-}\left(\rho_{\alpha}\left(g_{n}\right)\right)$ with $\left\|v_{+}\right\|>1-3 \rho_{n}^{\prime}$. Then

By the triangle inequality,

$$
\frac{\rho_{\alpha}\left(g_{n}\right)}{\lambda_{1}\left(\rho_{\alpha}\left(g_{n}\right)\right)}\left(v_{\xi}\right)=v_{+}+\frac{\rho_{\alpha}\left(g_{n}\right)}{\lambda_{1}\left(\rho_{\alpha}\left(g_{n}\right)\right)}\left(v_{<}\right)
$$

$$
\left\|v_{+}\right\|-\left\|\frac{\rho_{\alpha}\left(g_{n}\right)}{\lambda_{1}\left(\rho_{\alpha}\left(g_{n}\right)\right)}\left(v_{<}\right)\right\| \leq \frac{\left\|\rho_{\alpha}\left(g_{n}\right) v_{\xi}\right\|}{\lambda_{1}\left(\rho_{\alpha}\left(g_{n}\right)\right)} \leq\left\|v_{+}\right\|+\left\|\frac{\rho_{\alpha}\left(g_{n}\right)}{\lambda_{1}\left(\rho_{\alpha}\left(g_{n}\right)\right)}\left(v_{<}\right)\right\| .
$$

Hence

$$
1-3 \rho_{n}^{\prime}-\left\|\frac{\rho_{\alpha}\left(g_{n}\right)}{\lambda_{1}\left(\rho_{\alpha}\left(g_{n}\right)\right)}\left(v_{<}\right)\right\| \leq \frac{\left\|\rho_{\alpha}\left(g_{n}\right) v_{\xi}\right\|}{\lambda_{1}\left(\rho_{\alpha}\left(g_{n}\right)\right)} \leq 1+\left\|\frac{\rho_{\alpha}\left(g_{n}\right)}{\lambda_{1}\left(\rho_{\alpha}\left(g_{n}\right)\right)}\left(v_{<}\right)\right\| .
$$

The eigenvalues of $\frac{\rho_{\alpha}\left(g_{n}\right)}{\lambda_{1}\left(\rho_{\alpha}\left(g_{n}\right)\right)}$ restricted to $X_{-}\left(g_{n}\right)$ are $\exp \left(\chi_{\alpha}\left(\lambda\left(g_{n}\right)\right)-\chi_{\max , \alpha}\left(\lambda\left(g_{n}\right)\right)\right)$, where $\chi_{\alpha} \neq \chi_{\max , \alpha}$ is a restricted weight of $\Sigma\left(\rho_{\alpha}\right)$. They converge to zero and these endomorphisms 
are all diagonalisable. Hence,

$$
\left\|\frac{\rho_{\alpha}\left(g_{n}\right) \mid X_{-}\left(g_{n}\right)}{\lambda_{1}\left(\rho_{\alpha}\left(g_{n}\right)\right)}\right\| \underset{n+\infty}{\rightarrow} 0
$$

Taking the logarithm and the upper bound of $\frac{\left\|\rho_{\alpha}\left(g_{n}\right) v_{\xi}\right\|}{\lambda_{1}\left(\rho_{\alpha}\left(g_{n}\right)\right)}$ and its inverse, we obtain for $n$ large enough,

$$
\left\|\sigma\left(g_{n}, \xi\right)-\sigma\left(g_{n}, g_{x} \eta_{0}\right)\right\| \leq 3 \rho_{n}^{\prime}+\sup _{\alpha \in \Pi}\left\|\frac{\rho_{\alpha}\left(g_{n}\right) \mid X_{-}\left(g_{n}\right)}{\lambda_{1}\left(\rho_{\alpha}\left(g_{n}\right)\right)}\right\| .
$$

Finally, for any $v \in \mathfrak{a}$, there exists $t_{n} \rightarrow+\infty, \varepsilon_{n}^{\prime} \rightarrow i d_{G}$, so that for any $n$ large enough,

$$
\left\|\lambda\left(\gamma_{n}\right)-\left(v+t_{n} \theta\right)\right\| \leq 3 \rho_{n}^{\prime}+\sup _{\alpha \in \Pi}\left\|\frac{\rho_{\alpha}\left(g_{n}\right)_{\mid X_{-}\left(g_{n}\right)}}{\lambda_{1}\left(\rho_{\alpha}\left(g_{n}\right)\right)}\right\|+C_{\varepsilon_{n}^{\prime}} .
$$

The three terms converge to zero when $n \rightarrow+\infty$, so that, for $n$ large enough, $\lambda\left(\gamma_{n}\right)-\left(v+t_{n} \theta\right)$ is uniformly bounded.

To conclude, recall that the limit cone is the smallest closed cone containing all the Jordan projections of $\Gamma$. Hence, this implies that for $n$ large enough, the distance $d\left(v+t_{n} \theta, \mathcal{C}(\Gamma)\right)$ is uniformly bounded. Now, assume by contradiction that $\theta$ is in the boundary of $\mathcal{C}(\Gamma)$. Let $H$ be a supporting hyperplane of the convex $\mathcal{C}(\Gamma)$ at $\theta$ and $H^{+}$the half space not containing $\mathcal{C}(\Gamma)$. Pick $v \in H^{+}$, whose distance to $\mathcal{C}(\Gamma)$ is large. Then $d\left(v+\mathbb{R}^{+} \theta, \mathcal{C}(\Gamma)\right)=d\left(v+\mathbb{R}^{+} \theta, H\right)=d(v, H)$ is also large. This is contradictory with inequality (8).

Hence, topological transitivity of the dynamical system $\left(\Omega(X), \phi_{\theta}^{t}\right)$ implies that $\theta \in \stackrel{\circ}{\mathcal{C}}(\Gamma)$.

\section{TOPOLOGICAL MiXING}

Recall the definition of topological mixing.

Definition 5.1. Fix a direction $\theta \in \mathfrak{a}_{1}^{++}$. The Weyl chamber flow $\phi_{\mathbb{R}}^{\theta}$ is topologically mixing on $\Omega(X)$ if for all open subsets $U, V \subset \Omega(X)$, there exists $T>0$ such that for all $t \geq T$, we have $U \cap \phi_{t}^{\theta}(V) \neq \emptyset$.

It will be sometimes more convenient to make proofs in the cover $\widetilde{\Omega}(X)$, where the topological mixing takes the following form : for all open subsets $\widetilde{U}, \widetilde{V} \subset \widetilde{\Omega}(X)$, there exists $T>0$ such that for all $t \geq T$ there exists $\gamma_{t} \in \Gamma$ with $\gamma_{t} \widetilde{U} \cap \phi_{t}^{\theta}(\widetilde{V}) \neq \emptyset$.

5.1. Non-arithmetic spectrum. Denote by $\Gamma^{l o x}$ the set of loxodromic elements of $\Gamma$. In [Dal00] Dal'bo introduced the notion of non-arithmetic spectrum for subgroup of $\operatorname{Isom}\left(H^{n}\right)$, meaning that the length spectrum of such a group is not contained in a discrete subgroup of $\mathbb{R}$.

We generalize this definition for isometry group in higher rank:

Definition 5.2. We say that $\Gamma$ has non-arithmetic spectrum if the length spectrum $\lambda\left(\Gamma^{\text {lox }}\right)$ spans a dense subgroup of $\mathfrak{a}$.

Proposition 5.3. Every Zariski dense semigroup $\Gamma$ contains loxodromic elements, strong $(r, \varepsilon)$ Schottky Zariski dense semigroups and has non-arithmetic spectrum.

Proof. For a general semisimple, connected, real linear Lie group, Benoist proves in [Ben00, Proposition 0] that when $\Gamma$ is a Zariski dense semigroup of $G$, then the additive group generated by the full length spectrum $\lambda(\Gamma)$ is dense in $\mathfrak{a}$. Thus, this Proposition implies that Zariski dense semigroups containing only loxodromic elements have non-arithmetic length spectrum. In particular, strong $(r, \varepsilon)$-Schottky Zariski dense semigroups have non-arithmetic length spectrum. Finally, the existence of Zariski dense Schottky semigroups in Zariski dense subgroups of $G$ follows from [Ben97, Proposition 4.3 for $\theta=\Pi]$. 
Prasad and Rapinchuk [PR05, Theorem 2] prove that every Zariski dense semigroup of $G$ contains a finite subset $F$ such that $\lambda(F)$ generates a dense subgroup of $\mathfrak{a}$.

5.2. A key proposition for mixing. The following proposition is the technical point for proving the topological mixing of the Weyl chamber flow. Roughly, it shows that among elements of $\Gamma$ which do not move too much a flat, (ie. $\left.\left(\gamma_{t}^{+}, \gamma_{t}^{-}\right) \in \mathcal{U}^{(2)}\right)$ for any given $x \in \mathfrak{a}$, we can find an element which send 0 to $x+\theta t$ for large $t$ (ie. $\lambda\left(\gamma_{t}\right) \in B(x+t \theta, \eta)$ )

Proposition 5.4. Fix $\theta \in \mathfrak{a}_{1}^{++}$in the interior of the limit cone $\mathcal{C}(\Gamma)$.

Then for every nonempty open subset $\mathcal{U}^{(2)} \subset L^{(2)}(\Gamma)$, for all $x \in \mathfrak{a}$ and $\eta>0$ there exists $T>0$ such that for all $t \geq T$ there exists a loxodromic element $\gamma_{t} \in \Gamma$ with

$$
\left\{\begin{array}{r}
\left(\gamma_{t}^{+}, \gamma_{t}^{-}\right) \in \mathcal{U}^{(2)} \\
\lambda\left(\gamma_{t}\right) \in B(x+t \theta, \eta)
\end{array}\right.
$$

We will need the following classical density lemma, see for example [Ben00, Lemma 6.2].

Lemma 5.5. Let $V$ be a real vector space of finite dimension. Let $l_{0}, l_{1}, \ldots, l_{t}$ be vectors of $V$ and $\eta>0$. Set

$$
L:=\sum_{0 \leq i \leq t} \mathbb{R}_{+} l_{i}, M:=\sum_{0 \leq i \leq t} \mathbb{Z} l_{i}, \text { and } M_{+}:=\sum_{0 \leq i \leq t} \mathbb{N} l_{i} .
$$

Assume that $M$ is $\eta$-dense in $V$. Then there exists $v_{0} \in V$ such that $M_{+}$is $\eta$-dense in $v_{0}+L$.

Remark that if $M_{+}$is $\eta$-dense in $v_{0}+L$ then it is $\eta$-dense in $v+L$ for every $v \in v_{0}+L$.

The following lemma is a consequence of [Ben97, Proposition 4.3].

Lemma 5.6. For all $\theta$ in the interior of the limit cone $\mathcal{C}(\Gamma)$, there exists a finite set $S \subset \Gamma, a$ positive number $r>0$ and $\varepsilon_{n} \underset{+\infty}{\rightarrow} 0$ such that

(i) $\theta$ is in the interior of the convex cone $L(S):=\sum_{\gamma \in S} \mathbb{R}_{+} \lambda(\gamma)$,

(ii) the elements of $\lambda(S)$ form a basis of $\mathfrak{a}$,

(iii) for all $n \geq 1$, the family $S_{n}:=\left(\gamma^{n}\right)_{\gamma \in S}$ spans a Zariski-dense strong $\left(r, \varepsilon_{n}\right)-$ Schottky semigroup of $\Gamma$.

Proof. Fix $\theta$ in the interior of $\mathcal{C}(\Gamma)$.

Let us now construct a family of $r_{G}$ open cones in the limit cone $\mathcal{C}(\Gamma)$. We consider a affine chart of $\mathbb{P}(\mathfrak{a})$ centered in $\mathbb{R} \theta$. Since $\mathbb{R} \theta$ is in the open set $\mathbb{P}(\stackrel{\mathcal{C}}{\mathcal{C}}(\Gamma))$, it admits an open, polygonal, convex neighborhood with $r_{G}$ distinct vertices centered in $\mathbb{R} \theta$ and included in $\mathbb{P}(\stackrel{\circ}{\mathcal{C}}(\Gamma))$. We denote by $p:=\left(\mathbb{R} p_{i}\right)_{1 \leq i<r_{G}}$ the family of vertices of that convex neighbourhood, $\mathscr{H}_{p}$ its convex hull. Without loss of generality we can assume that there exists $\delta_{0}>0$ so that the $\delta_{0}-$ neighbourhood of $\mathscr{H}_{p}, \mathcal{V}_{\delta_{0}}\left(\mathscr{H}_{p}\right)$ is included in $\mathbb{P}(\stackrel{\circ}{\mathcal{C}}(\Gamma))$.

For any $\delta>0$, we denote by $\mathcal{V}_{\delta}\left(\partial \mathscr{H}_{p}\right)$ the $\delta$-neighborhood of the boundary $\partial \mathscr{H}_{p}$. Choose $0<\delta \leq \inf \left(\delta_{0}, \frac{1}{3} d\left(\mathbb{R} \theta, \partial \mathscr{H}_{p}\right)\right)$ so that $\mathbb{R} \theta \in \mathscr{H}_{p} \backslash \mathcal{V}_{\delta}\left(\mathscr{H}_{p}\right)$

Denote by $L_{p} \subset \stackrel{\circ}{\mathcal{C}}(\Gamma)$ (resp. $\left.\mathcal{V}_{\delta}\left(\partial L_{p}\right)\right)$ the closed (resp. open) cone whose projective image is $\mathscr{H}_{p}\left(\right.$ resp. $\left.\mathcal{V}_{\delta}\left(\partial \mathscr{H}_{p}\right)\right)$. For all $1 \leq i \leq r_{G}$, denote by $\left(\Omega_{i}\right)_{1 \leq i \leq r_{G}}$ the family of open cones such that $\mathbb{P}\left(\Omega_{i}\right):=B_{\mathbb{P}(\mathfrak{a})}\left(p_{i}, \delta\right)$.

By [Ben97, Proposition 4.3] applied to the finite family of disjoint open cones $\left(\Omega_{i}\right)_{1 \leq i \leq r_{G}}$ there exists $0<\varepsilon_{0} \leq r$, a generating set $S:=\left\{\gamma_{i}\right\}_{1 \leq i \leq r_{G}} \subset \Gamma$ of a Zariski dense $(r, \varepsilon)$-Schottky semigroup such that for all $1 \leq i \leq r_{G}$ the Jordan projection $\lambda\left(\gamma_{i}\right)$ is in $\Omega_{i}$. By Lemma 3.3, for any $n \geq 1$, the elements of $S_{n}$ are $\left(r, \varepsilon_{n}\right)$-loxodromic. Thus, for $n$ large, condition (iii) holds. By construction, $\lambda(S)$ form a family of $r_{G}$ linearly independent vectors of $\mathfrak{a}$ hence (ii) holds. Set 
$L(S):=\sum_{\gamma \in S} \mathbb{R}_{+} \lambda(\gamma)$. The construction of $L_{p}$ and $\mathcal{V}_{\delta}\left(\partial L_{p}\right)$ implies that $\theta \in L_{p} \backslash \mathcal{V}_{\delta}\left(\partial L_{p}\right)$. Since $\lambda\left(\gamma_{i}\right) \in \Omega_{i} \subset \mathcal{V}_{\delta}\left(\partial L_{p}\right)$ for all $1 \leq i \leq r_{G}$, the boundary of the cone $\partial L(S) \subset \mathcal{V}_{\delta}\left(\partial L_{p}\right)$. Hence $L_{p} \backslash \mathcal{V}_{\delta}\left(\partial L_{p}\right) \subset \stackrel{\circ}{L}(S)$ and finally, condition (i) holds, $\theta$ is in the interior of the cone $L(S)$.

Let us give a proof of the key Proposition.

Proof of Proposition 5.4. We fix a point $\theta$ in the interior of $\mathcal{C}(\Gamma)$, an open, nonempty set $\mathcal{U}=$ $\mathcal{U}^{+} \times \mathcal{U}^{-} \subset L^{(2)}(\Gamma)$, a point $x \in \mathfrak{a}$ and $\eta>0$.

Consider $S$ as in the previous Lemma 5.6. Denote by $\Gamma_{n}$ the semigroup spanned by $S^{n}$.

By Lemma 3.7, one can pick $h \in \Gamma^{l o x}$ such that $\left(h^{+}, h^{-}\right) \in \mathcal{U}^{(2)} \backslash\left(\gamma_{1}^{-}, \gamma_{r_{G}}^{+}\right)$. Choose $r>0$ so that

$$
r \leq \inf \left(\rho, \frac{1}{6} d\left(h^{+}, h^{-}\right), \frac{1}{6} d\left(\gamma_{r_{G}}^{+}, h^{-}\right), \frac{1}{6} d\left(h^{+}, \gamma_{1}^{-}\right)\right) .
$$

In particular, Proposition 3.17 holds for elements of the form $h \gamma_{r_{G}}^{n_{r_{G}}} g \gamma_{1}^{n_{1}} h$ where $g \in \Gamma_{n}$.

Choose $0<\varepsilon \leq r$ small enough so that

$$
\left\{\begin{array}{r}
\left(3 r_{G}+2\right) C_{r, \varepsilon} \leq \eta / 2 \\
B\left(h^{+}, \varepsilon\right) \times B\left(h^{-}, \varepsilon\right) \subset \mathcal{U}^{(2)}
\end{array}\right.
$$

where $\left(C_{r, \varepsilon}\right)_{\varepsilon \geq 0}$ are constants given by the Proposition.

We use Lemma 3.3 and choose $n$ large so that $h^{n}, S^{n}$ are $\left(r, \varepsilon_{n}\right)$-loxodromic elements with $\varepsilon_{n} \leq \varepsilon$.

By Proposition 5.3, the subgroup generated by $\lambda\left(\Gamma_{n}\right)$ is dense in $\mathfrak{a}$. By Lemma 6.1 applied to $\lambda\left(\Gamma_{n}\right)$, there exists a finite subset $F \subset \Gamma_{n}$ containing at most $2 r_{G}$ elements so that $\lambda\left(S^{n}\right) \cup \lambda(F)$ spans a $\eta / 2$-dense subgroup of $\mathfrak{a}$. We denote by $l$ the number of elements in $S^{\prime}:=S^{n} \cup F$ and we enumerate the elements of $S^{n} \cup F$ by $\left(g_{1}, \ldots, g_{l}\right)$, where $g_{1}:=\gamma_{1}^{n}$ and $g_{l}:=\gamma_{r_{G}}^{n}$. A crucial fact is that $l \leq 3 r_{G}$ is bounded independently of $\lambda\left(\Gamma_{n}\right)$.

The additive subgroup generated by $\lambda\left(S^{\prime}\right)$ is $\eta / 2$-dense in $\mathfrak{a}$. Furthermore, $\theta$ is still in the interior of the convex cone $L\left(S^{\prime}\right):=\sum_{g \in S^{\prime}} \mathbb{R}_{+} \lambda(g)$ by (i). Lemma 5.5 gives the existence of $v_{0} \in \mathfrak{a}$ such that $M_{+}\left(S^{\prime}\right):=\sum_{g \in S^{\prime}} \mathbb{N} \lambda(g)$ is $\eta / 2-$ dense in $v_{0}+L\left(S^{\prime}\right)$.

The interior of $L\left(S^{\prime}\right)$ contains $\theta$. Hence for any $v \in \mathfrak{a}$, the intersection $\left(v+\mathbb{R}_{+} \theta\right) \cap\left(v_{0}+L\left(S^{\prime}\right)\right)$ is a half line.

Consider such a half line $x-\nu\left(h^{n}, g_{l}, \ldots, g_{1}, h^{n}\right)-2 \lambda\left(h^{n}\right)+\theta[T,+\infty)$ contained in $v_{0}+L\left(S^{\prime}\right)$, for some $T \in \mathbb{R}$. For all $t \geq T$, there exists $n_{t}:=\left(n_{t}(1), \ldots, n_{t}(l)\right) \in \mathbb{N}^{l}$ such that

$$
\left\|\sum_{i=1}^{l} n_{t}(i) \lambda\left(g_{i}\right)-x+\nu\left(h^{n}, g_{l}, \ldots, g_{1}, h^{n}\right)+2 \lambda\left(h^{n}\right)-\theta t\right\| \leq \eta / 2 .
$$

Furthermore, Proposition 3.17 applied to $\gamma_{t}:=h^{n} g_{l}^{n_{t}(l)} \ldots g_{1}^{n_{t}(l)} h^{n}$ gives

$$
\left\|\lambda\left(\gamma_{t}\right)-\sum_{i=1}^{l} n_{t}(i) \lambda\left(g_{i}\right)-2 \lambda\left(h^{n}\right)-\nu\left(h^{n}, g_{l}, \ldots, g_{1}, h^{n}\right)\right\| \leq(l+2) C_{r, \varepsilon}
$$

and $\left(\gamma_{t}^{+}, \gamma_{t}^{-}\right) \in B\left(h^{+}, \varepsilon\right) \times B\left(h^{-}, \varepsilon\right) \subset \mathcal{U}^{(2)}$ by $(10)$.

Finally, we have $\left(3 r_{G}+2\right) C_{r, \varepsilon} \leq \eta / 2$ by the choice of $n, S^{n}, h^{n}$. Once again, remark it is necessary for $l$ to be bounded independently of $\Gamma$ and $n$. We get the following bound using the triangle inequality,

$$
\left\|\lambda\left(\gamma_{t}\right)-x-\theta t\right\| \leq \eta
$$

This concludes the proof. 
Note that it is possible to use the density [PR05, Theorem 2] of Prasad and Rapinchuk instead of our density Lemma 6.1. Start by following our proof, choose $S \subset \Gamma$ as in the Lemma 5.6. Use now Prasad and Rapinchuk's density Theorem, there is a finite subset $F$ of the semigroup generated by $S$ so that $\langle\lambda(F)\rangle$ is dense in $\mathfrak{a}$. Remark that for any $n \in \mathbb{N}$, the subset $S_{n}^{\prime \prime}:=F^{n} \cup S^{n}$ is finite, has at most $|F|+r_{G}$ elements and the subgroup generated by $\lambda\left(S_{n}^{\prime \prime}\right)$ is also dense in $\mathfrak{a}$. It suffices then to follow the end of the proof by taking $S^{\prime}=S_{n}^{\prime \prime}$ for $n$ large enough so that $S^{\prime}$ is a $\left(r, \varepsilon_{n}\right)$-Schottky semigroup with $\left(|F|+r_{G}+2\right) C_{r, \varepsilon_{n}} \leq \eta / 2$.

5.3. Proof of the main Theorem 1.2. We end the proof of the main theorem with Proposition 5.4 and Theorem 4.5 .

Proof of Theorem 1.2. If $\left(\Omega(X), \phi_{t}^{\theta}\right)$ is topologically mixing, it is in particular topologically transitive. Therefore by Proposition 4.7 if $\left(\Omega(X), \phi_{t}^{\theta}\right)$ is topologically mixing $\theta$ is in the interior of the limit cone.

Let us prove that if $\theta \in \stackrel{\circ}{\mathcal{C}}(\Gamma) \cap \mathfrak{a}_{1}^{++}$then $\left(\Omega(X), \phi_{t}^{\theta}\right)$ is topologically mixing.

Let $\widetilde{U}, \widetilde{V}$ be two open subsets of $\widetilde{\Omega}(X)$. Without loss of generality, we can assume that $\widetilde{U}=\mathcal{H}^{-1}\left(\mathcal{U}^{(2)} \times B(u, r)\right)\left(\right.$ resp. $\left.\widetilde{V}=\mathcal{H}^{-1}\left(\mathcal{V}^{(2)} \times B(v, r)\right)\right)$ where $\mathcal{U}^{(2)}$ and $\mathcal{V}^{(2)}$ are open subsets of $L^{(2)}(\Gamma)$, and $B(u, r), B(v, r)$ open balls of $\mathfrak{a}$.

Recall that for all $g \in \Gamma$, using Hopf coordinates

$$
\left\{\begin{array}{r}
\mathcal{H}^{(2)}\left(g\left(\mathcal{U}^{(2)}\right) \times B(u, r)\right)=g \mathcal{U}^{(2)} \\
\left.\mathcal{H}\left(\phi_{t}^{\theta}\left(\mathcal{V}^{(2)}\right) \times B(v, r)\right)\right)=\mathcal{V}^{(2)} \times B(v+\theta t, r)
\end{array}\right.
$$

We begin by transforming the coordinates in $L^{(2)}(\Gamma)$ to recover the setting of Proposition 5.4. By Theorem 4.5, there exists $g \in \Gamma$ such that $g \mathcal{U}^{(2)} \cap \mathcal{V}^{(2)} \neq \emptyset$. For such an element $g \in \Gamma$, the subset $g \mathcal{U}^{(2)} \cap \mathcal{V}^{(2)}$ is a nonempty open subset of $L^{(2)}(\Gamma)$. Let $\mathcal{O}^{(2)}:=\mathcal{O}_{+} \times \mathcal{O}_{-} \subset g \mathcal{U}^{(2)} \cap \mathcal{V}^{(2)}$ be a nonempty open subset, such that $r:=d\left(\overline{\mathcal{O}_{+}}, \overline{\mathcal{O}_{-}}\right)>0$.

Remark that $g \widetilde{U} \cap\left(\mathcal{H}^{(2)}\right)^{-1}\left(\mathcal{O}^{(2)}\right)$ is open and non empty. Thus it contains an open box $\mathcal{H}^{-1}\left(\mathcal{O}^{(2)} \times B\left(u^{\prime}, r^{\prime}\right)\right)$ with $u^{\prime} \in \mathfrak{a}$ and $r^{\prime}>0$. Set $\eta:=\min \left(r, r^{\prime}\right)$.

By Proposition 5.4 applied to $\mathcal{O}^{(2)}, x=v-u^{\prime} \in \mathfrak{a}$ and $\eta>0$, there exists $T>0$ such that for all $t \geq T$ there exists $\gamma_{t} \in \Gamma$ with

$$
\left\{\begin{array}{r}
\left(\gamma_{t}^{+}, \gamma_{t}^{-}\right) \in \mathcal{O}^{(2)} \\
\lambda\left(\gamma_{t}\right) \in B\left(v-u^{\prime}+t \theta, \eta\right)
\end{array}\right.
$$

Remark that every loxodromic element $\gamma \in \Gamma$ fixes its limit points in $L^{(2)}(\Gamma)$. Thus for all such $\gamma \in \Gamma$ with $\left(\gamma^{+}, \gamma^{-}\right) \in \mathcal{O}^{(2)}$, the subset $\gamma \mathcal{O}^{(2)} \cap \mathcal{O}^{(2)}$ is open and non empty (it contains $\left.\left(\gamma^{+}, \gamma^{-}\right)\right)$. Furthermore, $\lambda(\gamma)=\sigma\left(\gamma, \gamma^{+}\right)$by Fact 2.7. Hence

$$
\left\{\begin{array}{r}
\gamma_{t} \mathcal{O}^{(2)} \cap \mathcal{O}^{(2)} \neq \emptyset \\
u^{\prime}+\sigma\left(\gamma_{t}, \gamma_{t}^{+}\right) \in B(v+t \theta, \eta)
\end{array}\right.
$$

The subset $\gamma_{t} g \tilde{U} \cap\left(\mathcal{H}^{(2)}\right)^{-1}\left(\gamma_{t} \mathcal{O}^{(2)} \cap \mathcal{O}^{(2)}\right)$ is open, non empty and contains the point of coordinates $\left(\gamma_{t}^{+}, \gamma_{t}^{-}, u^{\prime}+\sigma\left(\gamma_{t}, \gamma_{t}^{+}\right)\right) \in \mathcal{H}^{-1}\left(\phi_{t}^{\theta}(\tilde{V})\right)$. Finally, $\gamma_{t} g \tilde{U} \cap \phi_{t}^{\theta}(\tilde{V}) \neq \emptyset$, as $\tilde{U}, \tilde{V}$ are arbitrary, it proves that $\phi_{t}^{\theta}$ is topological mixing.

\section{Appendix: A DENSITY LEMma}

The following density lemma is crucial for the proof of proposition 5.4. 
Lemma 6.1. Let $d \in \mathbb{N}$, let $V$ be a real vector space of dimension $d$. For all $E \subset V$ that spans a dense additive subgroup of $V$, for all $\epsilon>0$, for any basis $B \subset E$ of $V$, there exists a finite subset $F \subset E$ of at most $2 d$ elements so that $B \cup F$ spans a $\epsilon$-dense additive subgroup of $V$.

Proof. We show the lemma by induction.

Let $\mathcal{E} \subset \mathbb{R}^{1}=V$ be a subset that generates a dense additive subgroup of $\mathbb{R}$. Let $x \in \mathbb{R}$ a basis, ie. a non zero element. Any element $y$ in $\mathcal{E}$ so that $\langle y, x\rangle$ is dense is a solution. We assume that $\mathcal{E}$ contains no such element. Consider the quotient $\mathbb{R} / x \mathbb{Z}$ and $p: \mathbb{R} \rightarrow \mathbb{R} / x \mathbb{Z}$ the projection. The set $\mathcal{E}$ projects to a infinite subset of $\mathbb{R} / x \mathbb{Z}$, therefore it has an accumulation point. Let $f_{1} \neq f_{2} \in \mathcal{E}$ be two elements such that $\left|p\left(f_{1}\right)-p\left(f_{2}\right)\right|<\epsilon$. Then $\left\langle x, f_{1}, f_{2},\right\rangle$, generates a $\epsilon-$ dense additive subgroup of $\mathbb{R}$, the Lemma is proved for $\operatorname{dim}(V)=1$, where $F=\left\{f_{1}, f_{2}\right\}$.

Now consider a vector space $V$ of dimension $d$. Let $\mathcal{E}$ be a a subset of $V$ such that $\overline{\langle\mathcal{E}\rangle}=V$ and $\mathcal{B}=\left(b_{1}, \ldots, b_{d}\right) \subset E$ a basis of $V$. Without loss of generality we suppose that the basis is the standard basis and the norm is the sup norm : these only affect computations up to a multiplicative constant.

Suppose that we have $f_{1}, f_{2} \in \mathcal{E}$ such that the additive group $\left\langle f_{1}, f_{2}, \mathcal{B}\right\rangle$ contains a non zero vector $u$ of norm $\|u\| \leq \epsilon / 2$. We will show that it is enough to conclude and then prove the existence of such elements.

Consider $V^{\prime}=u^{\perp}$, the decomposition $V=u \oplus V^{\prime}$ and $p^{\prime}$ the projection on $V^{\prime}$. Let $\mathcal{E}^{\prime}=p^{\prime}(\mathcal{E})$ and $\mathcal{B}^{\prime}$ a basis of $V^{\prime}$ included in $p^{\prime}(\mathcal{B})$. By induction, there is a finite subset $\mathcal{F}^{\prime} \subset \mathcal{E}^{\prime}$ of at most $2(d-1)$ elements such that $\left\langle\mathcal{F}^{\prime}, \mathcal{B}^{\prime}\right\rangle$ generates an $\epsilon / 2$-dense additive subgroup of $V^{\prime}$. For all $f^{\prime} \in \mathcal{F}^{\prime}$ there is $f \in \mathcal{E}$ and $\lambda_{f} \in \mathbb{R}$ such that $f^{\prime}=f+\lambda_{f} u$. A similar result holds for elements of $\mathcal{B}^{\prime}$. We denote by $\mathcal{F} \subset \mathcal{E}$ a choice of lifts for elements of $\mathcal{F}^{\prime}$. We claim that the set $F=\mathcal{F} \cup\left\{f_{1}, f_{2}\right\} \cup \mathcal{B}$ generates a $\epsilon$-dense additive subgroup of $V$.

Let $x \in V, x=x^{\prime}+\lambda_{x} u$. By hypothesis, there is $\left(n_{f^{\prime}}\right)_{f^{\prime} \in \mathcal{F}^{\prime}} \in \mathbb{Z}^{\left|\mathcal{F}^{\prime}\right|}$, and $\left(n_{b^{\prime}}\right)_{b^{\prime} \mathcal{B}^{\prime}} \in \mathbb{Z}^{d-1}$ and $\alpha \in V^{\prime}$ satisfying $\left\|\alpha^{\prime}\right\|<\epsilon / 2$ such that :

$$
x^{\prime}=\sum_{f^{\prime} \in \mathcal{F}^{\prime}} n_{f^{\prime}} f^{\prime}+\sum_{b^{\prime} \in \mathcal{B}^{\prime}} n_{b^{\prime}} b^{\prime}+\alpha^{\prime} .
$$

Therefore,

$$
x^{\prime}=\sum_{f \in \mathcal{F}} n_{f^{\prime}} f+\sum_{b \in \mathcal{B}} n_{b} b+\left(\sum_{f \in \mathcal{F}} n_{f^{\prime}} \lambda_{f}+\sum_{b \in \mathcal{B}} n_{b} \lambda_{b}\right) u+\alpha^{\prime} .
$$

Finally we get :

$$
x=\sum_{f \in \mathcal{F}} n_{f^{\prime}} f+\sum_{b \in \mathcal{B}} n_{b} b+[k] u+(k-[k]) u+\alpha^{\prime}
$$

where $k=\left(\sum_{f \in \mathcal{F}} n_{f^{\prime}} \lambda_{f}+\sum_{b \in \mathcal{B}} n_{b}+\lambda_{x}\right)$ and $[k] \in \mathbb{Z}$ denotes the integer part of $k$. The vector $\sum_{f \in \mathcal{F}} n_{f^{\prime}} f+\sum_{b \in \mathcal{B}} n_{b} b+[k] u$ is in the additive group generated by $F$ and $|(k-[k]) u+\alpha| \leq \epsilon$. This proves the claim.

To finish the proof we need to show that for any $\epsilon>0$, there are elements $f_{1}, f_{2} \in \mathcal{E}$ such that $\left\langle f_{1}, f_{2}, \mathcal{B}\right\rangle$ contains a non zero vector of norm less than $\epsilon$.

Consider the natural projection $p: \mathbb{R}^{d} \rightarrow \mathbb{R}^{d} / \oplus_{k=1}^{d} \mathbb{Z} b_{k}$ into the torus $\mathbb{R}^{d} / \oplus_{k=1}^{d} \mathbb{Z} b_{k}$. If there is an element $f \in \mathcal{E}$ so that $p(\mathbb{Z} f)$ contains accumulation points, we choose $u$, non zero and small in $\langle\mathcal{B}, f\rangle$. We assume now that there is no such element in $E$. Choose an integer $N$ so that $N>\frac{2 \sqrt{d}}{\varepsilon}$. By the pigeon hole principle on $N^{d}+1$ distinct elements of $\mathcal{E}$, we deduce the existence of $f_{1}, f_{2} \in \mathcal{E}$ with $0<\left|p\left(f_{1}-f_{2}\right)\right|<\frac{\epsilon}{2}$. The unique representative of the projection $p\left(f_{1}-f_{2}\right)$ in the fundamental domain $\sum_{i=1}^{d}(0,1] b_{i}$ is a suitable choice for $u$. Indeed, it is an element of the subgroup $\left\langle f_{1}, f_{2}, \mathcal{B}\right\rangle$ and it is of norm at most $\frac{\epsilon}{2}$. 


\section{REFERENCES}

[Ben97] Yves Benoist. Propriétés asymptotiques des groupes linéaires. Geometric \&3 Functional Analysis GAFA, 7(1):1-47, 1997.

[Ben00] Yves Benoist. Propriétés asymptotiques des groupes linéaires ii. Advanced Studies Pure Math., 26:33-48, 2000.

[BG03] Emmanuel Breuillard and Tsachik Gelander. On dense free subgroups of lie groups. Journal of Algebra, 261(2):448-467, 2003.

[BQ16] Yves Benoist and Jean-François Quint. Random walks on reductive groups. In Random Walks on Reductive Groups, pages 153-167. Springer, 2016.

[CG02] Jean-Pierre Conze and Yves Guivarc'h. Densité d'orbites d'actions de groupes linéaires et propriétés d'équidistribution de marches aléatoires. In Rigidity in dynamics and geometry, pages 39-76. Springer, 2002.

[Dal00] Françoise Dal'bo. Topologie du feuilletage fortement stable. Ann. Inst. Fourier (Grenoble), 50(3):981993, 2000.

[Ebe72] Patrick Eberlein. Geodesic flows on negatively curved manifolds i. Annals of Mathematics, pages 492510, 1972.

[GJT12] Yves Guivarc'h, Lizhen Ji, and John C Taylor. Compactifications of symmetric spaces, volume 156. Springer Science \& Business Media, 2012.

[Hel78] Sigurdur Helgason. Differential geometry and symmetric spaces, volume 12. Academic press, 1978.

[Kim06] Inkang Kim. Length spectrum in rank one symmetric space is not arithmetic. Proceedings of the American Mathematical Society, 134(12):3691-3696, 2006.

[PR05] Gopal Prasad and Andrei S Rapinchuk. Zariski-dense subgroups and transcendental number theory. Mathematical Research Letters, 12(2):239-248, 2005.

[Qui02] Jean-François Quint. Divergence exponentielle des sous-groupes discrets en rang supérieur. Commentarii Mathematici Helvetici, 77(3):563-608, 2002.

[Sam15] Andres Sambarino. The orbital counting problem for hyperconvex representations [sur le décompte orbital pour les representations hyperconvexes]. In Annales de l'institut Fourier, volume 65, pages 17551797, 2015

[Ser16] Çağri Sert. Joint Spectrum and Large Deviation Principles for Random Matrix Products. PhD thesis, Paris-Saclay, Université, 2016.

[Thi07] Xavier Thirion. Sous-groupes discrets de $S L(d, R)$ et equidistribution dans les espaces symetriques. $\mathrm{PhD}$ thesis, Tours, 2007.

[Thi09] Xavier Thirion. Propriétés de mélange du flot des chambres de weyl des groupes de ping-pong. Bull. Soc. Math. France, 137(3):387-421, 2009.

[Tit71] Jacques Tits. Représentations linéaires irréductibles d'un groupe réductif sur un corps quelconque. Journal für die reine und angewandte Mathematik, 247:196-220, 1971. 